\title{
Review Article \\ Recent Advances in Graphene-Assisted Nonlinear Optical Signal Processing
}

\author{
Jian Wang and Xiao Hu \\ Wuhan National Laboratory for Optoelectronics, School of Optical and Electronic Information, \\ Huazhong University of Science and Technology, Wuhan, Hubei 430074, China
}

Correspondence should be addressed to Jian Wang; jwang@hust.edu.cn

Received 25 December 2015; Accepted 24 February 2016

Academic Editor: Xueqing Zhang

Copyright (C) 2016 J. Wang and X. Hu. This is an open access article distributed under the Creative Commons Attribution License, which permits unrestricted use, distribution, and reproduction in any medium, provided the original work is properly cited.

Possessing a variety of remarkable optical, electronic, and mechanical properties, graphene has emerged as an attractive material for a myriad of optoelectronic applications. The wonderful optical properties of graphene afford multiple functions of graphene based polarizers, modulators, transistors, and photodetectors. So far, the main focus has been on graphene based photonics and optoelectronics devices. Due to the linear band structure allowing interband optical transitions at all photon energies, graphene has remarkably large third-order optical susceptibility $\chi^{(3)}$, which is only weakly dependent on the wavelength in the nearinfrared frequency range. The graphene-assisted four-wave mixing (FWM) based wavelength conversions have been experimentally demonstrated. So, we believe that the potential applications of graphene also lie in nonlinear optical signal processing, where the combination of its unique large $\chi^{(3)}$ nonlinearities and dispersionless over the wavelength can be fully exploited. In this review article, we give a brief overview of our recent progress in graphene-assisted nonlinear optical device and their applications, including degenerate FWM based wavelength conversion of quadrature phase-shift keying (QPSK) signal, phase conjugated wavelength conversion by degenerate FWM and transparent wavelength conversion by nondegenerate FWM, two-input and three-input highbase optical computing, and high-speed gate-tunable terahertz coherent perfect absorption (CPA) using a split-ring graphene.

\section{Introduction}

Graphene, a monolayer of carbon atoms arranged in a twodimensional honeycomb lattice, is the building block of the familiar graphite. In 2004 [1], a research team based in Manchester successfully isolated graphene by mechanical exfoliation. Since then, many extraordinary properties have been reported, such as extremely high charge-carrier mobility $200,000 \mathrm{~cm}^{2} \mathrm{~V}^{-1} \mathrm{~s}^{-1}$. Graphene possesses linear, massless band structure $E_{+}(p)= \pm V|p|$, where the upper (lower) sign corresponds to the electron (hole) band, $p$ is the quasimomentum, and $V \approx 10^{6} \mathrm{~m} / \mathrm{s}$ is the Fermi velocity. The high mobility of charge carriers and zero bandgap of graphene can be employed as an ideal medium for high-frequency applications, such as radio-frequency switches [2].

The photonic properties of graphene are equally remarkable. On one hand, many breakthroughs in researches on graphene, including ultrafast photodetectors [3], broadband polarizers [4], and modulators [5], stem from its unique band structure. The graphene electroabsorption modulator [5] is based on interband transitions which can be tuned by applying drive voltage, correspondingly changing the Fermi energy $\left(E_{F}\right)$ of graphene. Here, it has to be emphasized that at short wavelengths range (i.e., infrared and visible), the graphene optical absorptions are determined by interband transitions, whereas, at long wavelengths range (i.e., terahertz), they are dominated by intraband transitions. In the mid- to farinfrared and $\mathrm{THz}$ ranges, graphene exhibits a strong plasmonic response. Doped and patterned graphene can support localized plasmonic resonances which significantly enhance the absorption [6]. On the other hand, the interband optical absorption in zero-gap graphene could be saturated readily from the visible to near-infrared region under strong excitation due to Pauli blocking [7]. Graphene could behave as a fast saturable absorber over a wide spectral range for the mode locking of fiber lasers. Since 2009, graphene based 
Interband



(a)

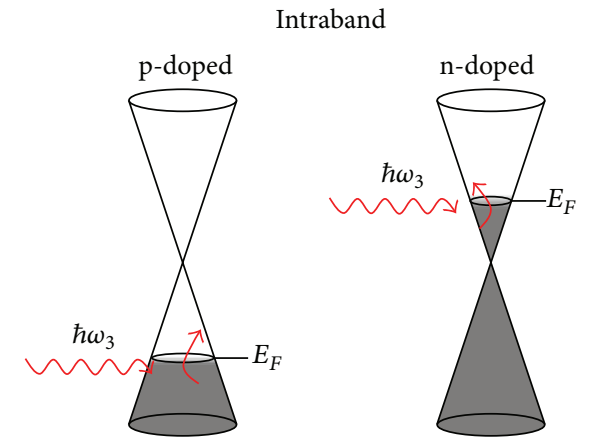

(b)

FIGURE 1: (a) Interband and (b) intraband absorption mechanisms in graphene.

mode-locked lasers have been applied to a wide variety of laser configurations and operational wavelengths [7-11]. Graphene has been suggested as a material that might have large $\chi^{(3)}$ nonlinearities, which is also due to its linear band structure allowing interband optical transitions at all photon energies. Reports in literature [12] indicate that the nonlinear response of graphene is essentially dispersionless over the wavelength and much stronger compared to bulk semiconductors. Zhang and coworkers have experimentally demonstrated the nonlinear refractive index of graphene to be as high as $n_{2} \approx 10^{-7} \mathrm{~cm}^{2} \mathrm{~W}^{-1}$ [13] by using Z-scan technique. After that, optical bistability, self-induced regenerative oscillations, and FWM have been consecutively observed in graphene-silicon hybrid optoelectronic devices [14]. FWM has also been demonstrated in graphene in various configurations, for example, slow-light graphene-silicon photonic crystal waveguide [15], graphene optically deposited onto fiber ferrules [16], and graphene-coated microfiber [17, 18]. Moreover, FWM based wavelength conversion of a $10-\mathrm{Gb} / \mathrm{s}$ non-return-to-zero (NRZ) signal with mechanically exfoliated graphene was first reported in [19]. Advanced optical modulation formats play an important role in enabling highcapacity optical transport networks [20] where wavelength conversion function is highly desired.

In this review article, we go over our recent progress in graphene-assisted nonlinear optical device and their applications, including degenerate FWM based tunable wavelength conversion of QPSK signal [23], phase conjugated wavelength conversion by degenerate FWM and transparent wavelength conversion by nondegenerate FWM, two-input optical highbase hybrid doubling and subtraction functions [24], threeinput high-base optical computing, and high-speed gatetunable terahertz coherent perfect absorption using a splitring graphene [25].

\section{Optical Properties of Graphene [26-28]}

The primary mechanism of optical absorption in graphene involves two processes: carrier intraband transitions and interband transitions. For short wavelengths (i.e., infrared and visible range), the graphene optical absorption is determined by interband transitions, whereas for long wavelengths (i.e., terahertz range) it is dominated by intraband transitions. Figure 1 pictorially depicts the basic mechanisms. Interband transitions refer to an exchange of charge carriers between the conduction and valence bands; the energy of a photon $\hbar \omega$ should be satisfying the relationship $\hbar \omega \geq 2 E_{F}$ as shown in Figure 1(a). In n-doped graphene, an optical photon $\left(\hbar \omega_{2}\right)$ with energy less than $2 E_{F}$ cannot be absorbed because the electron states in resonance in the conduction band are occupied. In p-doped graphene, an optical photon $\left(\hbar \omega_{2}\right)$ with energy less than $2 E_{F}$ cannot be absorbed because there are no electrons available for the interband transition. For the low frequency $\mathrm{THz}$ range ( $\hbar \omega \leq 2 E_{F}$ ), the intraband absorption mechanism is shown in Figure 1(b). And graphene behaves like a conductive film, and its optical conductivity closely follows its electrical conductivity, which can be described by a simple Drude model.

For the optical conductivity of graphene, an analytic expression derived within the random phase approximation [29] is used, which is

$$
\begin{aligned}
\sigma= & \sigma_{\text {inter }}+\sigma_{\text {intra }}=\frac{i 8 \sigma_{0}}{\pi} \frac{E_{\text {th }}}{E_{\mathrm{ph}}+i E s} \ln \left[2 \cos \left(\frac{E_{F}}{2 E_{\text {th }}}\right)\right] \\
& +\sigma_{0}\left[\frac{1}{2}+\frac{1}{\pi} \tan ^{-1}\left(\frac{E_{\mathrm{ph}}-2 E_{F}}{2 E_{\mathrm{th}}}\right)\right. \\
& \left.-\frac{i}{2 \pi} \ln \frac{\left(E_{\mathrm{ph}}+2 E_{F}\right)^{2}}{\left(E_{\mathrm{ph}}-2 E_{F}\right)^{2}+4 E_{\mathrm{th}}^{2}}\right],
\end{aligned}
$$

where $\sigma_{0}=e^{2} / 4 \hbar$ is the universal conductivity of graphene ( $e$ is the charge of an electron and $\hbar$ is the reduced Planck constant), $E_{\text {th }}=k_{B} T$ is thermal energy in $\mathrm{eV}\left(k_{B}\right.$ is the Boltzmann constant and $T$ is the temperature), $E_{F}$ is the Fermi energy (i.e., chemical potential $\mu$ ) of graphene in $\mathrm{eV}$, $E_{\mathrm{ph}}=h c / \lambda$ is photon energy in $\mathrm{eV}$ ( $h$ is the Planck constant), and $E_{s}=\hbar / \tau$ is scattering energy in $\mathrm{eV}$ for the scattering time $\tau$. 


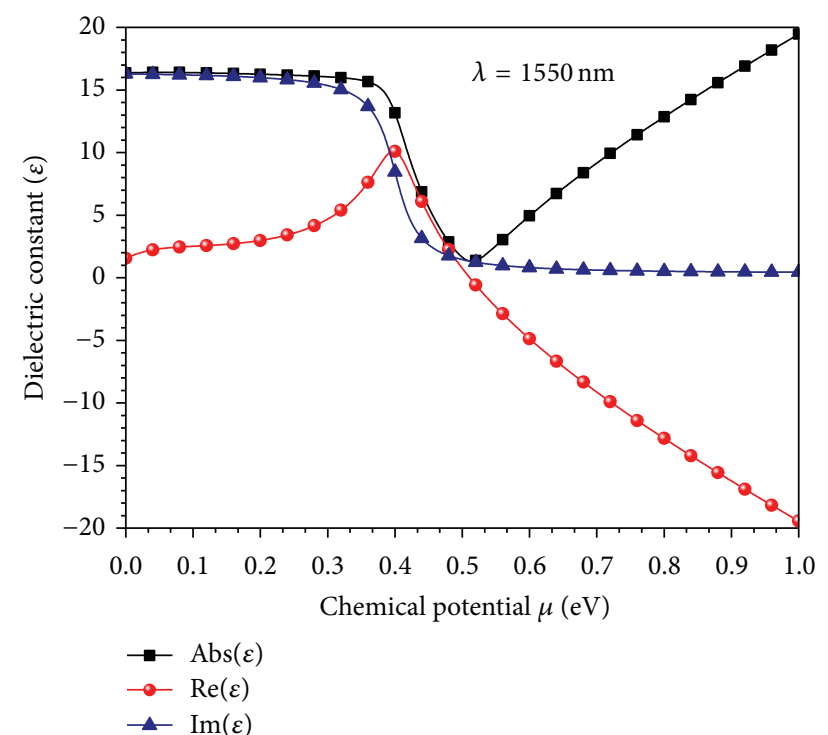

FIGURE 2: Calculated dielectric constant of graphene (real part, imaginary part, and magnitude) as a function of chemical potential $\mu$ of graphene for $\lambda=1550 \mathrm{~nm}$.

The complex dielectric function $\varepsilon(\mu)$ can be obtained from the complex optical conductivity of graphene written by

$$
\varepsilon(\mu)=2.5+\frac{i \sigma(\mu)}{\omega \varepsilon_{0} \Delta},
$$

where $\Delta=0.7 \mathrm{~nm}$ is a thickness of the graphene layer and $\varepsilon_{0}$ is the permittivity of vacuum. The dielectric constant $\varepsilon$ of graphene is calculated as a function of the chemical potential $\mu$ for a wavelength of $\lambda=1550 \mathrm{~nm}, T=300 \mathrm{~K}, \lambda=1550 \mathrm{~nm}$, and $\tau=0.1$ ps, as shown in Figure 2 .

Due to the linear band structure of graphene allowing interband optical transitions at all photon energies, graphene has been suggested as a material that might have large $\chi^{(3)}$ nonlinearities. In [12] the principle of graphene based degenerate FWM is proposed. Two continuous-wave (CW) pumps with frequencies $\omega_{1}$ and $\omega_{2}$ are combined together in graphene based device with high third-order nonlinearity $\left(\chi^{(3)}\right)$ and mixed together to generate a new coherent beam with frequency $\omega_{\text {con }}$ (converted idler), as depicted in Figure 3.

In the near and far-IR and terahertz ranges $(0.1-10 \mathrm{THz})$, doped graphene has recently emerged as a powerful plasmonic material $[6,27,30]$. Additionally, graphene offers the unique capability of plasmon tunability which can be realized by gated injection of charge carriers. As mentioned above, in terahertz range, the intraband optical transitions dominate. Based on random phase approximation [31-33], the complex conductivity of graphene can be described by the Drude model as $\sigma(\omega)=i e^{2} E_{F} / \pi \hbar^{2}\left(\omega+i \tau^{-1}\right)$, especially in heavily doped region and low frequencies (far below Fermi energy), where $E_{F}(\mu)$ depends on the concentration of charged doping and $\tau=\mu_{0} E_{F} / e \nu_{F}^{2}$, where $\nu_{F}$ is the Fermi velocity and $\omega, \mu_{0}$, and $e$ are the frequency, the dc mobility, and electron charge, respectively.

\section{Degenerate FWM Based Tunable Wavelength Conversion of QPSK Signal [23]}

Monolayer graphene was grown by the chemical vapor deposition (CVD) method [34]. Graphene was primarily grown on $\mathrm{Cu}$ foils $(25-\mu \mathrm{m}$ thick with a purity of $>99.99 \mathrm{wt} \%$ obtained from Alfa Aesar) in a hot wall furnace. The growth process can be briefly summarized as follows: (1) load the fused silica tube with the $\mathrm{Cu}$ foil, evacuate, backfill with hydrogen, heat to $1050^{\circ} \mathrm{C}$, and maintain a $\mathrm{H}_{2}(\mathrm{~g})$ pressure of 42 mTorr under a $2.5 \mathrm{sccm}$ flow; (2) stabilize the $\mathrm{Cu}$ film at the desired temperature, up to $1050^{\circ} \mathrm{C}$, and introduce $40 \mathrm{sccm}$ of $\mathrm{CH}_{4}(\mathrm{~g})$ for a desired period of time at a total pressure of 450 mTorr; (3) after exposure to $\mathrm{CH}_{4}$, cool the furnace to room temperature. Then, poly(methyl methacrylate) (PMMA) film was spin coated on the surface of the graphenedeposited $\mathrm{Cu}$ foil and the $\mathrm{Cu}$ foil was etched away with $1 \mathrm{M}$ $\mathrm{FeCl}_{3}$ solution. The resultant PMMA/graphene film $(5 \mathrm{~mm} \times$ $5 \mathrm{~mm}$ ) was washed in deionized water several times and transferred to deionized water solution or $\mathrm{Si} / \mathrm{SiO}_{2}$ substrate. Then, the floating PMMA/graphene sheet was mechanically transferred onto the fiber pigtail cross section and dried in a cabinet. After drying at room temperature for about 24 hours, the carbon atoms could be self-assembled onto the fiber endfacet thanks to the strong viscosity of graphene. The PMMA layer was finally removed by boiling acetone. By connecting this graphene-on-fiber component with another clean and dry FC/PC fiber connector, the nonlinear optical device was thereby constructed for FWM based wavelength conversion applications (Figure 4).

Optical image was taken with an optical microscope (Olympus DX51), and Raman spectroscopy was performed with a laser micro-Raman spectrometer (Renishaw inVia, $532 \mathrm{~nm}$ excitation wavelength). Scanning electron microscopy (SEM) images were obtained by Hitachi-S4800. The optical microscope (OM) image of the grown graphene film transferred on a $300 \mathrm{~nm} \mathrm{SiO}_{2} / \mathrm{Si}$ substrate was depicted in Figure 5(a). The grown graphene sheet was also transferred on silicon-on-insulator (SOI) for SEM characterization as shown in Figure 5(b). OM and SEM images shown in Figures 5(a) and 5(b) provided evidence of the uniformity of the graphene. Selected Raman spectrum was shown in Figure 5(c). Strong 2D and G bands were observed, accompanied by a weak $D$ band, at 2698,1582 , and $1351 \mathrm{~cm}^{-1}$, respectively. The $I_{2 \mathrm{D}} / I_{\mathrm{G}}$ ratio of 1.65 demonstrated the formation of monolayer graphene [34]. The low $\mathrm{D}$ to $\mathrm{G}$ peak intensity ratio $\sim 0.08$ indicates that the graphene formed on $\mathrm{SiO}_{2} / \mathrm{Si}$ substrate was almost defect-free $[35,36]$.

Figure 6 shows the experimental setup for degenerate FWM based wavelength conversion using a single-layer graphene grown by the CVD method. The CW output from an external cavity laser (ECL1) serves as the signal light for the degenerate FWM and is modulated with QPSK signal at 10 Gbaud by a single-polarization optical I/Q modulator. 


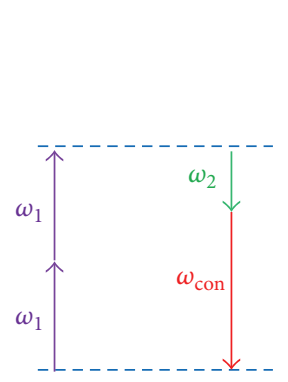

(a)

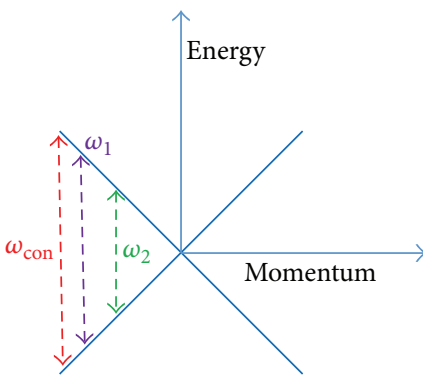

(b)

FIGURE 3: (a) Diagram of energy conservation in the degenerate FWM process. (b) Band structure of graphene with the three resonant photon energies (arrows) involved in degenerate FWM.

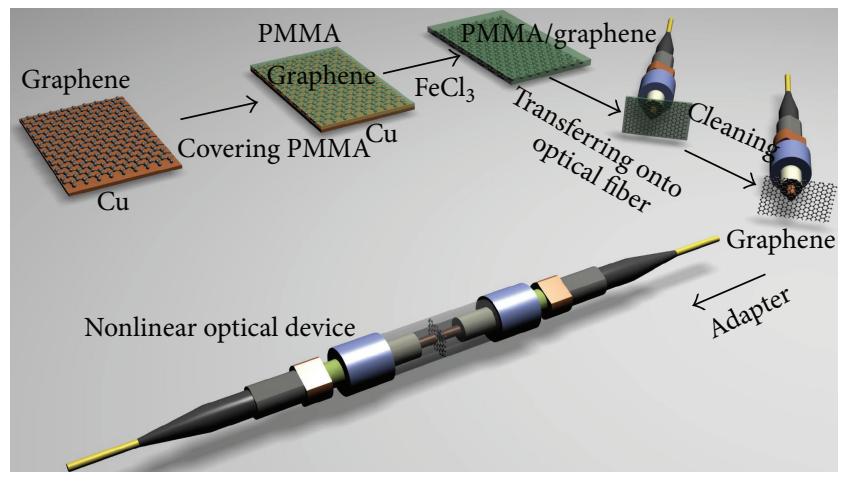

FIGURE 4: Fabrication process of the graphene-assisted nonlinear optical device.

An arbitrary waveform generator (AWG) running at $10 \mathrm{GS} / \mathrm{s}$ sampling rate is used to produce the electrical signal. The modulated 10-Gbaud QPSK signal is then amplified by an erbium-doped optical fiber amplifier (EDFA) followed by a thin film filter to suppress the amplified spontaneous emission (ASE) noise. Afterwards, the 10-Gbaud QPSK signal is combined with another CW light from ECL2 which serves as the pump light through a $3 \mathrm{~dB}$ coupler, amplified using a high-power EDFA (HP-EDFA), and launched into the singlelayer graphene sample. The polarization states of the CW pump and QPSK signal are adjusted to achieve optimized conversion efficiency of degenerate FWM in graphene. The amplified CW pump and QPSK signal take part in the degenerate FWM process when passing through the single-layer graphene sample and a newly converted idler is generated. After the FWM wavelength conversion, the converted idler is selected using two tunable filters (TF1, TF2) for coherent detection. First, the converted idler is selected using TF1. Since the power level of the converted idler is relatively low, the selected converted idler is amplified by EDFA2. Second, in order to suppress the amplified spontaneous emission (ASE) noise originated from EDFA2, another TF2 is employed. Hence, TF1 is used to select the converted idler, and TF2 is used to suppress the ASE noise. The CW output from ECL3 serves as a reference light for coherent detection. A variable optical attenuator (VOA) and a low noise EDFA (EDFA3) are employed to adjust the received signal-to-noise ratio (OSNR) for bit-error rate (BER) measurements. The optical spectra at different taps in the experimental setup are monitored by use of an optical spectrum analyzer (OSA).

In the experiment, the signal wavelength is fixed at $1550.12 \mathrm{~nm}$. Variable converted idler wavelength can be achieved simply by tuning the pump wavelength. Figure $7(\mathrm{a})$ shows a typical output FWM spectrum obtained after the CVD single-layer graphene-coated fiber device. A newly converted idler at $1546.88 \mathrm{~nm}$ is generated when the pump is tuned at $1548.49 \mathrm{~nm}$. We also measure the output spectrum without graphene for reference under the same experimental conditions. Moreover, we repeat the experiment by adding extra $2 \mathrm{~m}$ and $5 \mathrm{~m}$ single-mode fibers in the setup and get almost the same experimental results. As clearly shown in the inset of Figure 7(a), the power of converted idler without graphene is observed to be $\sim 5.5 \mathrm{~dB}$ lower than the one with graphene. That is, under the same experimental conditions, the converted idler without graphene is $\sim 71.9 \%$ lower than the one with graphene. Hence, the degenerate FWM in graphene contributes more to the wavelength conversion process. The insets of Figure 7(a) also depict measured QPSK constellations of the converted idler and the input signal. The background noise on the weak converted idler mainly causes the performance deterioration of the converted idler.

We define the conversion efficiency as the power ratio of converted idler to signal. We present a detailed comparison of the FWM conversion efficiency as a function of the pump power with and without graphene. As shown in Figure 7(b), the pump wavelength is fixed at $\lambda_{\text {pump }}=1548.49 \mathrm{~nm}$ and the signal is $\lambda_{\text {signal }}=1550.12 \mathrm{~nm}$. One can clearly see that the conversion efficiency increases with the pump power. When the pump power varies from $23 \mathrm{dBm}$ to $33 \mathrm{dBm}$, the enhanced FWM conversion efficiency by graphene changes from $4.7 \mathrm{~dB}$ to $7.5 \mathrm{~dB}$. In particular, the conversion efficiency curve in Figure 7 (b) seems to follow a 2:1 ratio for the conversion efficiency versus pump at relatively high pump power above $25 \mathrm{dBm}$, while going subquadratic at relatively lower pump power. Such interesting phenomenon might be ascribed to the saturable absorption effect of graphene. At lower pump power, the absorption by graphene limits the conversion 


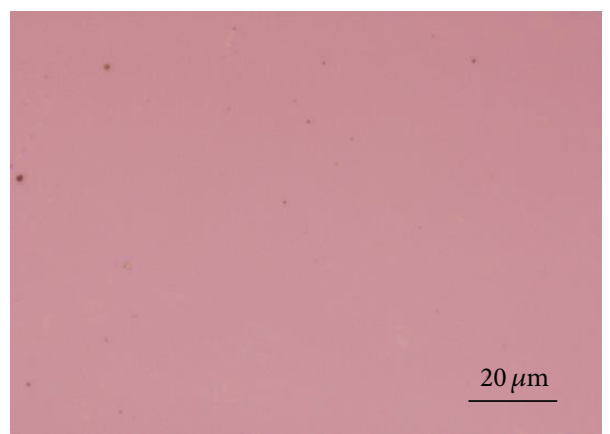

(a)

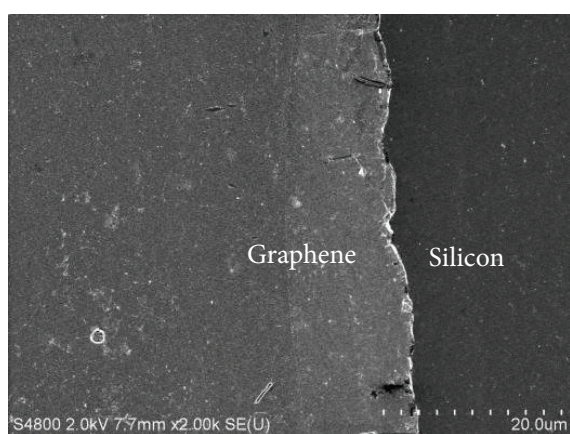

(b)

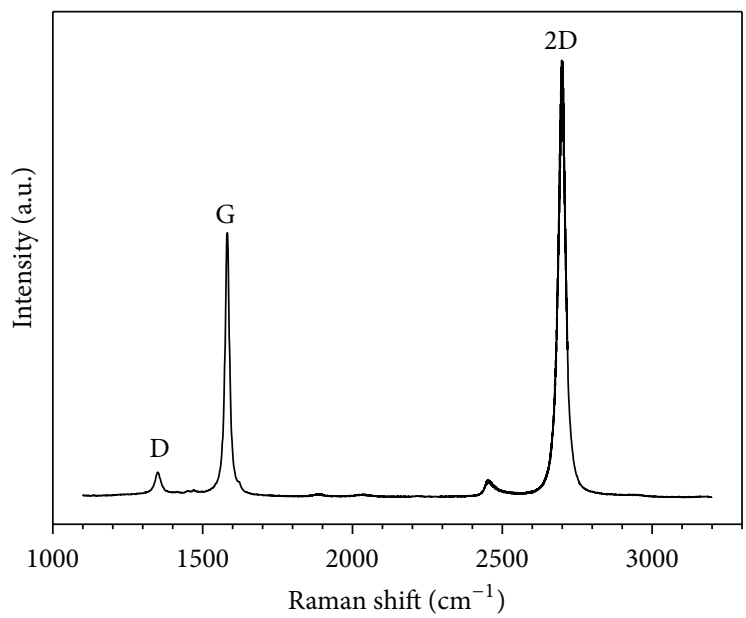

(c)

Figure 5: (a) Optical microscope (OM) image of graphene transferred on a $\mathrm{SiO}_{2} / \mathrm{Si}$ substrate. (b) Scanning electron microscope (SEM) image of graphene transferred on silicon-on-insulator (SOI). (c) Typical Raman spectrum of single-layer graphene on a $\mathrm{SiO}_{2} / \mathrm{Si}_{\mathrm{substrate}}(\mathrm{excitation}$ wavelength: $532 \mathrm{~nm}$ ).



FIGURE 6: Experimental setup for degenerate FWM based wavelength conversion in graphene. Inset: "sandwiched structure" graphene sample used as a nonlinear optical device. ECL: external cavity laser; AWG: arbitrary waveform generator; TF: tunable filter; OC: optical coupler; PC: polarization controller; OSA: optical spectrum analyzer; and VOA, variable optical attenuator.

efficiency, resulting in the subquadratic relationship between conversion efficiency and pump power. In contrast, at higher pump power, the absorption by graphene is saturable, and therefore the pump power dependent conversion efficiency follows a quadratic relationship which is in accordance with the theory of nonlinear coupled-mode equations under slowly varying envelope approximation and pump nondepletion approximation.

To characterize the performance of QPSK wavelength conversion, we further measure the BER curve as a function 




(a)

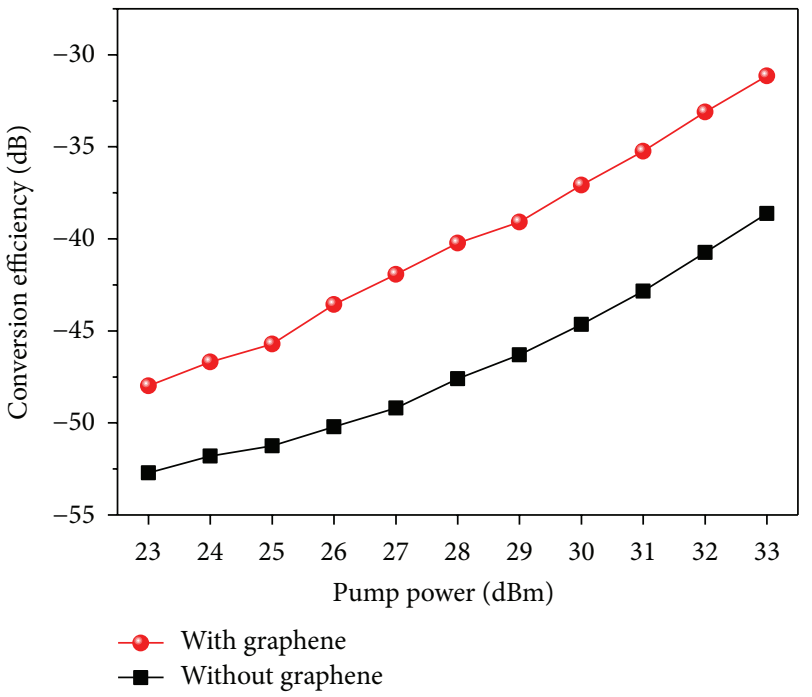

(b)

FIGURE 7: (a) Measured FWM spectra with (red) and without (black) graphene. (b) Measured conversion efficiency of FWM with and without graphene when pump power is tuned from $23 \mathrm{dBm}$ to $33 \mathrm{dBm}$.

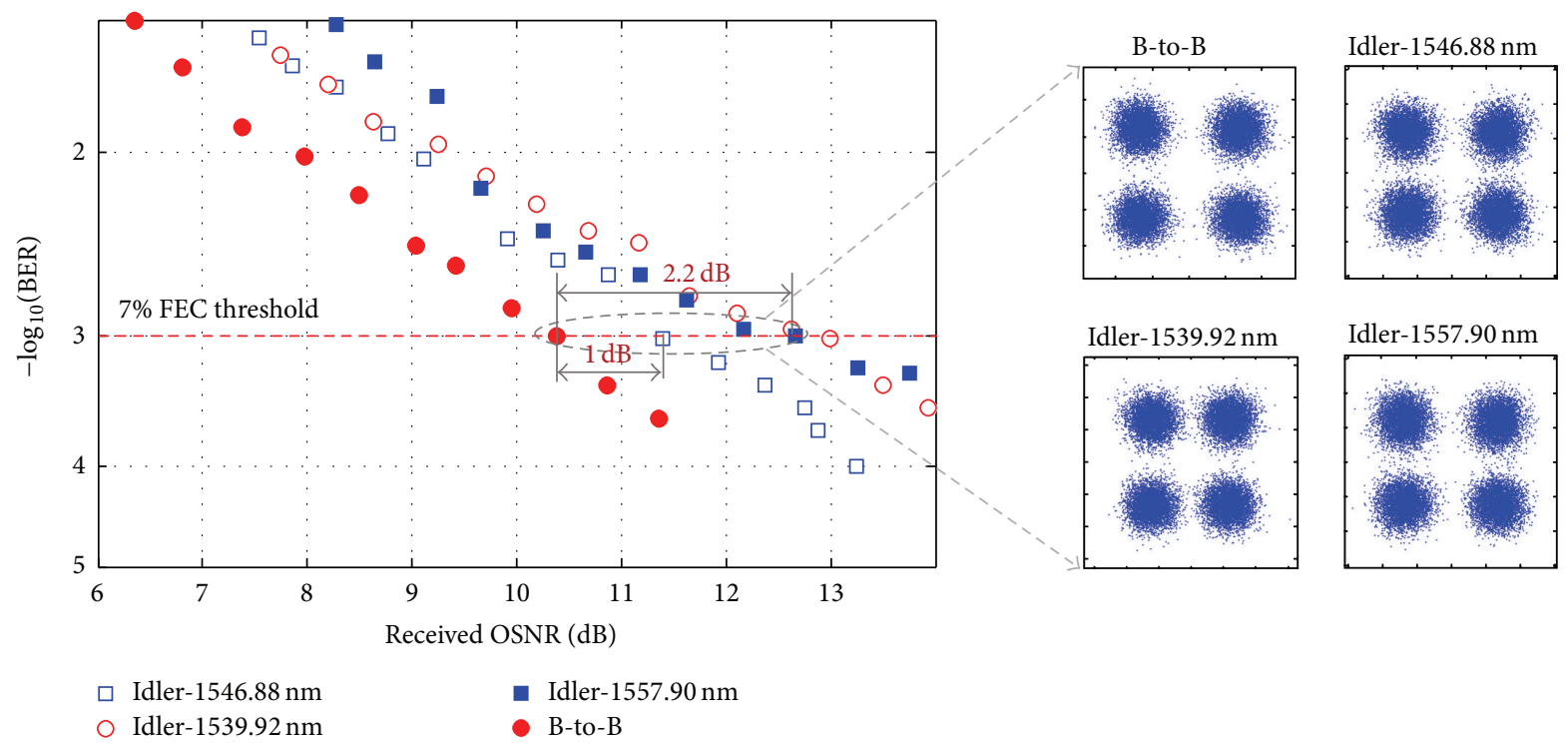

FIGURE 8: Measured BER versus received OSNR for wavelength conversion of QPSK signal. Insets show constellations of QPSK.

of the received OSNR for back to back (B-to-B) signal and converted idler. Figure 8 plots measured BER performance for tunable QPSK wavelength conversion with the converted idler generated at $1546.88,1539.92$, and $1557.90 \mathrm{~nm}$, respectively. The power of HP-EDFA is estimated to be $31 \mathrm{dBm}$. The measured conversion efficiencies for converted idlers at $1546.88,1539.92$, and $1557.90 \mathrm{~nm}$ are $-36.2,-48.2$, and $-39.8 \mathrm{~dB}$, respectively. As shown in Figure 8, the observed OSNR penalty is around $1 \mathrm{~dB}$ at a BER of $1 \times 10^{-3}(7 \%$ forward error correction (FEC) threshold) for QPSK wavelength conversion with the converted idler at $1546.88 \mathrm{~nm}$. The received OSNR penalties of $\sim 2.2 \mathrm{~dB}$ at a BER of $1 \times 10^{-3}$ are observed for converted idlers at 1539.92 and $1557.90 \mathrm{~nm}$.
The increased OSNR penalty is mainly due to the reduced conversion efficiency for converted idlers at 1539.92 and $1557.90 \mathrm{~nm}$. The right insets of Figure 8 depict corresponding constellations of the B-to-B signals and converted idlers. The OSNR penalty is believed to be originated from the relatively low conversion efficiency $(<-35 \mathrm{~dB})$, which can be ascribed to the very limited interaction between the singlelayer graphene and the propagating light only at the end face of optical fiber. With further improvement, one might enhance the conversion efficiency by mechanically transferring graphene sample grown by CVD method onto the $\mathrm{D}$ shaped fiber or microfiber to ensure more direct graphenelight interaction. 


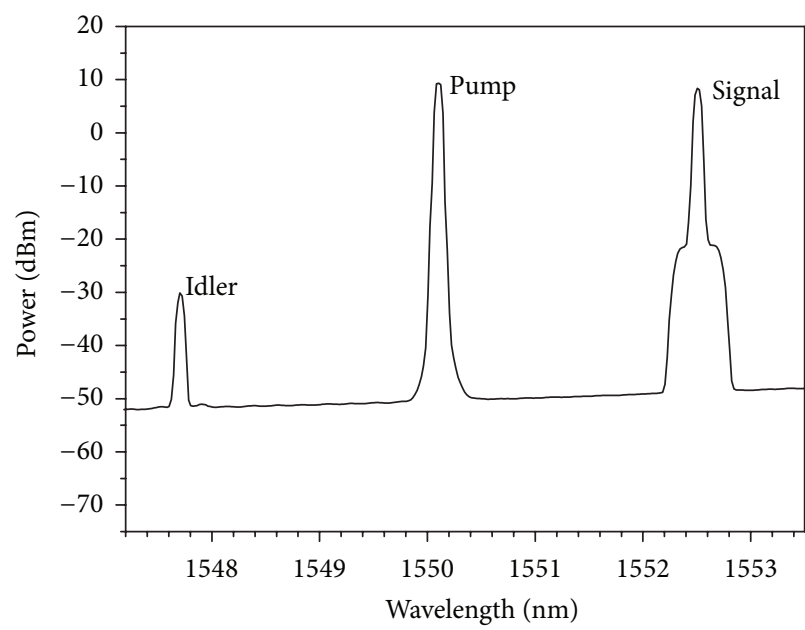

(a)

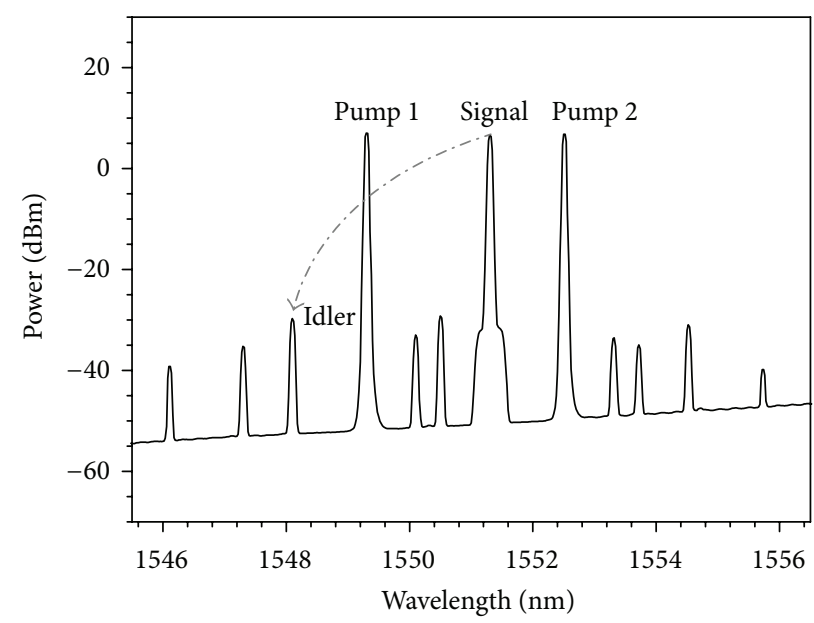

(b)

FIGURE 9: Measured (a) degenerate and (b) nondegenerate FWM spectrum with graphene-coated fiber device.

\section{Phase Conjugated and Transparent Wavelength Conversions of Nyquist 16-QAM Signals}

In this work, we show an experimental observation of degenerate/nondegenerate FWM based wavelength conversion of a 5-Gbaud Nyquist 16-ary quadrature amplitude modulation (16-QAM) signal, as Nyquist pulse shaping signals have been widely used in wavelength-division multiplexing (WDM) systems. Moreover, Nyquist WDM technology can transmit a number of different wavelength channels in a single fiber and exhibit higher spectrum efficiency in contrast with conventional WDM.

For the wavelength conversion based on degenerate FWM process as shown in Figure 9(a), the electric field of the newly converted idler meets the relationship of $E_{\text {idler }} \propto$ $E_{\text {pump }}^{2} E_{\text {signal }}^{*}$, where $E_{\text {idler }}, E_{\text {pump }}$, and $E_{\text {signal }}$ represent the complex electric fields of newly converted idler, input pump, and input signal, respectively. "*” denotes the complex conjugate of the electric field. Hence, the newly converted idler does not take the same data information carried by the original signal but its "phase conjugated" copy. For the wavelength conversion based on nondegenerate FWM process as shown in Figure 9(b), the electric field of the newly converted idler satisfies the relationship of $E_{\text {idler }} \propto$ $E_{\text {pump1 }} E_{\text {signal }} E_{\text {pump2 }}^{*}$, where $E_{\text {pump1 }}$ and $E_{\text {pump2 }}$ represent the complex electric fields of two input pumps. Therefore, the newly converted idler copies exactly the same data information carried by the original signal, that is, fully transparent wavelength conversion.

In order to verify the phase conjugated wavelength conversion by degenerate FWM and transparent wavelength conversion by nondegenerate FWM, we measure, record, and compare the typical time-varying symbol sequence of newly converted idlers by degenerate/nondegenerate FWM and original signal (B-to-B), as shown in Figure 10. One can clearly see from Figure 10 that the newly converted


FIGURE 10: Time-varying symbol sequence of newly converted idlers by degenerate/nondegenerate FWM and original signal (B-to-B).

idler by degenerate FWM flips its constellation points in the complex $I / Q$ plane with respect to the $I$-axis, corresponding to the phase conjugation of original Nyquist 16-QAM signal. In contrast, the newly converted idler by nondegenerate FWM duplicates the constellation of the original signal, corresponding to the transparent wavelength conversion of original Nyquist 16-QAM signal.

\section{Two-Input Optical High-Base Hybrid Doubling and Subtraction Functions [24]}

Figure 11 illustrates the concept and principle of two-input hybrid quaternary arithmetic functions. From the constellation in the complex plane (Figure 11(a)), it is clear that one can use four-phase levels $(\pi / 4,3 \pi / 4,5 \pi / 4,7 \pi / 4)$ of (D)QPSK 


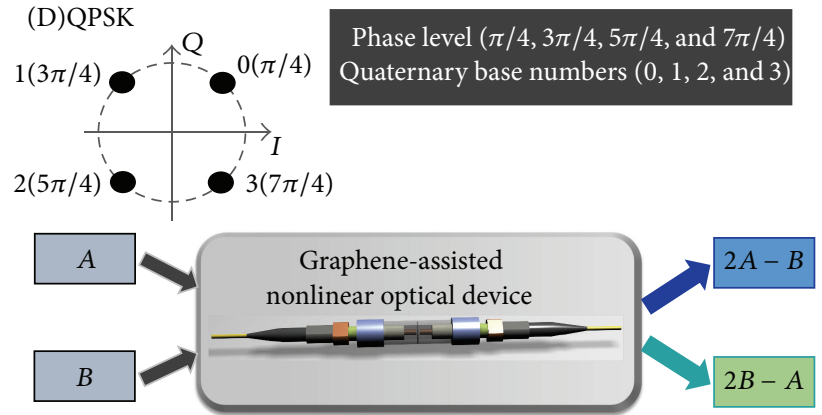

(a)

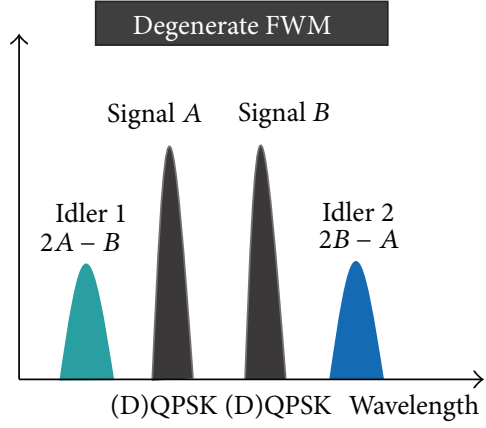

(b)

FIGURE 11: (a) Concept and (b) principle of hybrid quaternary arithmetic functions $(2 A-B, 2 B-A)$ using degenerate FWM and (D)QPSK signals.

to represent quaternary base numbers $(0,1,2,3)$. To implement two-input hybrid quaternary arithmetic functions, the aforementioned graphene-assisted nonlinear optical device is employed. Two-input quaternary numbers $(A, B)$ are coupled into the nonlinear device; then two converted idlers (idler 1, idler 2) are simultaneously generated by two degenerate FWM processes. Figure 9(b) illustrates the degenerate FWM process. We derive the electrical field $(E)$ and optical phase $(\phi)$ relationships of two degenerate FWM processes under the pump nondepletion approximation expressed as

$$
\begin{aligned}
& E_{i 1} \propto E_{A} \cdot E_{A} \cdot E_{B}^{*}, \\
& \phi_{i 1}=\phi_{A}+\phi_{A}-\phi_{B}, \\
& E_{i 2} \propto E_{B} \cdot E_{B} \cdot E_{A}^{*}, \\
& \phi_{i 2}=\phi_{B}+\phi_{B}-\phi_{A},
\end{aligned}
$$

where the subscripts $A, B, i 1$, and $i 2$ denote input signal $A$, signal $B$, converted idler 1 , and idler 2 , respectively. Owing to the phase wrap characteristic with a periodicity of $2 \pi$, it is implied from the linear phase relationships in (3) that idler 1 and idler 2 carry out modulo 4 operations of hybrid quaternary arithmetic functions of doubling and subtraction $(2 A-B, 2 B-A)$.

Figure 12 depicts measured typical spectrum obtained from the CVD single-layer graphene-coated fiber device. Two 10-Gbaud NRZ-(D)QPSK signals at $1550.10(A)$ and $1553.60 \mathrm{~nm}(B)$ are employed as two inputs. The power of two input signals $(A, B)$ is about $32 \mathrm{dBm}$. The conversion efficiency is measured to be around $-36 \mathrm{~dB}$. One can clearly see that two converted idlers are obtained by two degenerate FWM processes with idler 1 at $1546.60 \mathrm{~nm}(2 A-B)$ and idler 2 at $1557.20 \mathrm{~nm}(2 B-A)$. The resolution of the measured spectrum is set to $0.02 \mathrm{~nm}$. The steps in the measured spectrum are actually the modulation sidebands of two NRZ(D)QPSK carrying signals. In order to verify the hybrid quaternary arithmetic functions, we measure the phase of symbol sequence for two input signals and two converted idlers, as shown in Figure 13. By carefully comparing the quaternary base numbers for two input signals and two converted

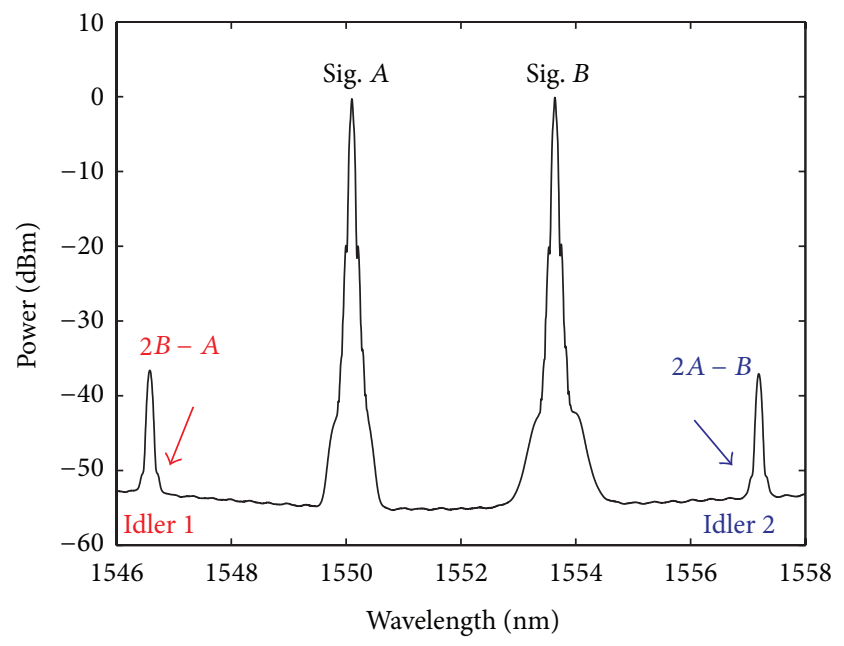

FIGURE 12: Measured spectrum for 10-Gbaud two-input hybrid quaternary arithmetic functions.

idlers, one can confirm the successful implementation of twoinput hybrid quaternary arithmetic functions of $2 A-B$ and $2 B-A$.

We further investigate the BER performance for the proposed optical two-input hybrid quaternary arithmetic functions. The observed OSNR penalties at a BER of $2 \times 10^{-3}$ for hybrid quaternary arithmetic functions are measured to be about 7.4 dB for $2 A-B$ and $7.0 \mathrm{~dB}$ for $2 B-A$. The insets in Figure 14(a) show constellations of the last point of the BER curves of output Sig. $B$ and $2 A-B$. The constellation of Sig. $B$ is measured under an OSNR of $12.6 \mathrm{~dB}$, while the constellation of $2 A-B$ is observed under an OSNR of $19.6 \mathrm{~dB}$. To clearly show the differences between these two constellations, we also assess the error vector magnitude (EVM) of these two constellations; that is, $\mathrm{EVM}=27.61 \%$ for output Sig. $B$ and EVM $=30.09 \%$ for output $2 A-B$. The significant performance degradations for the two-input hybrid quaternary arithmetic functions $(2 A-B, 2 B-A)$ might be ascribed to the relatively low conversion efficiency for two converted idlers at $1546.60 \mathrm{~nm}$ and $1557.20 \mathrm{~nm}$ and 

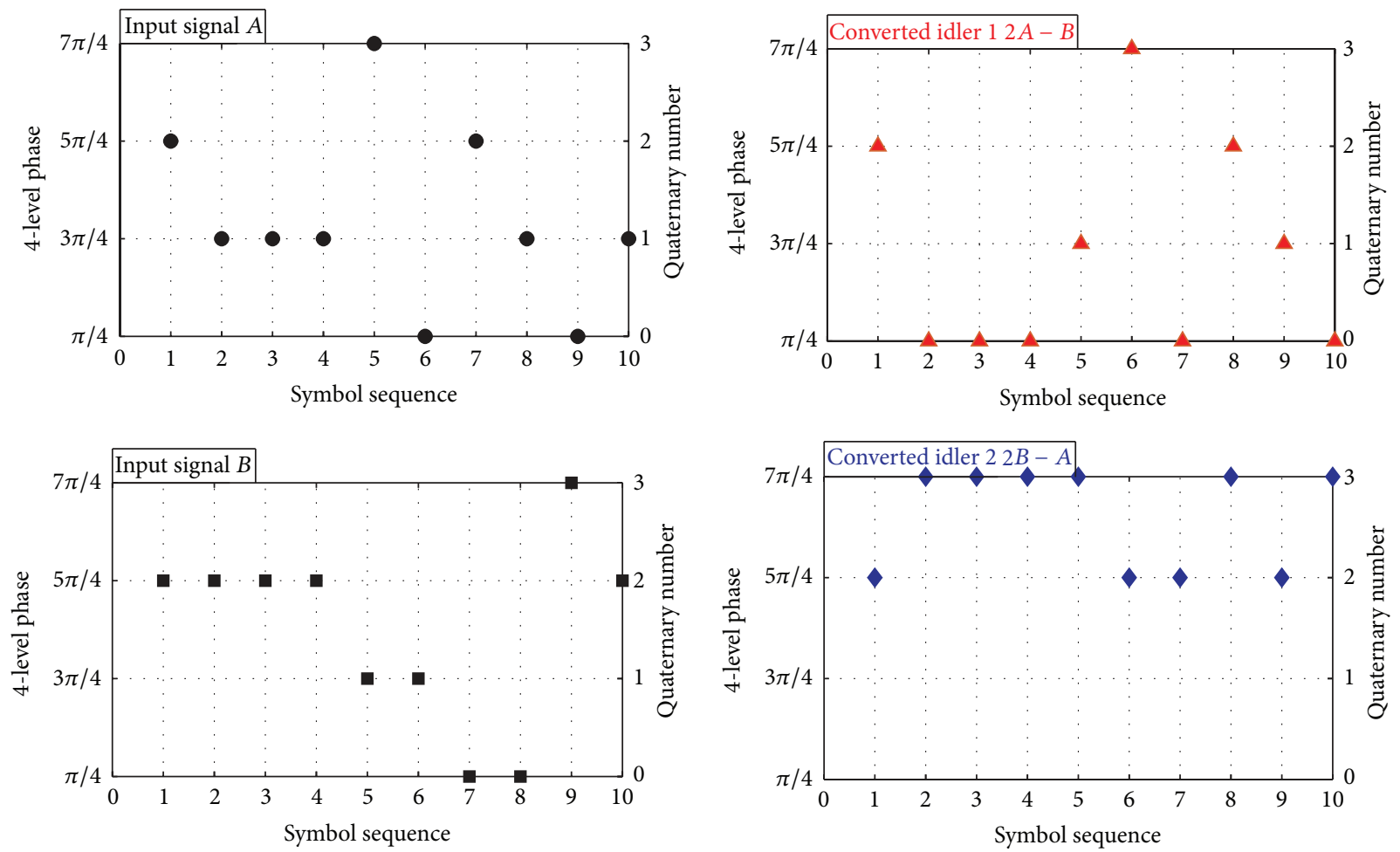

FIGURE 13: Measured phase of symbol sequence with coherent detection for 10-Gbaud two-input hybrid quaternary arithmetic functions.

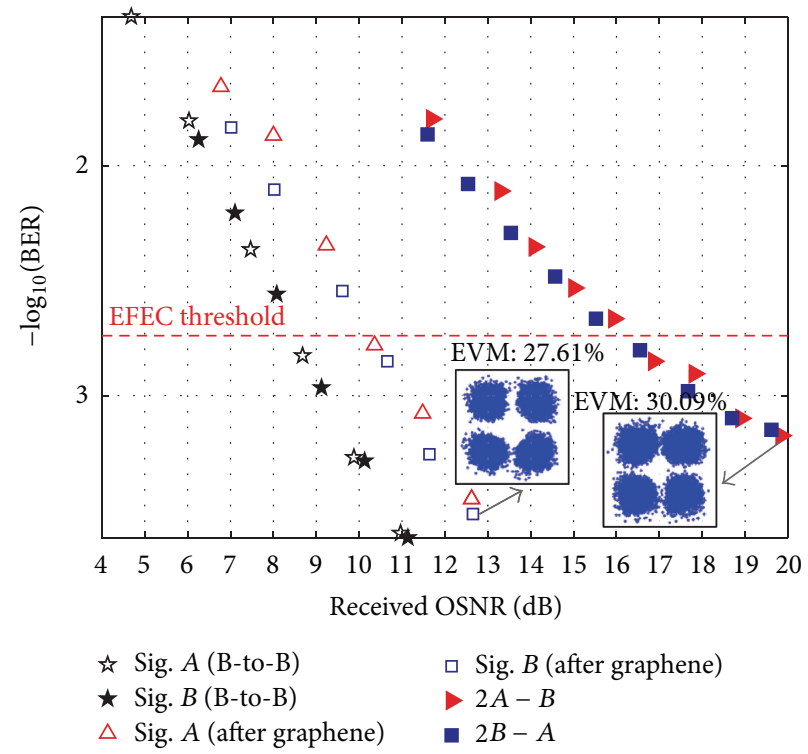

(a)

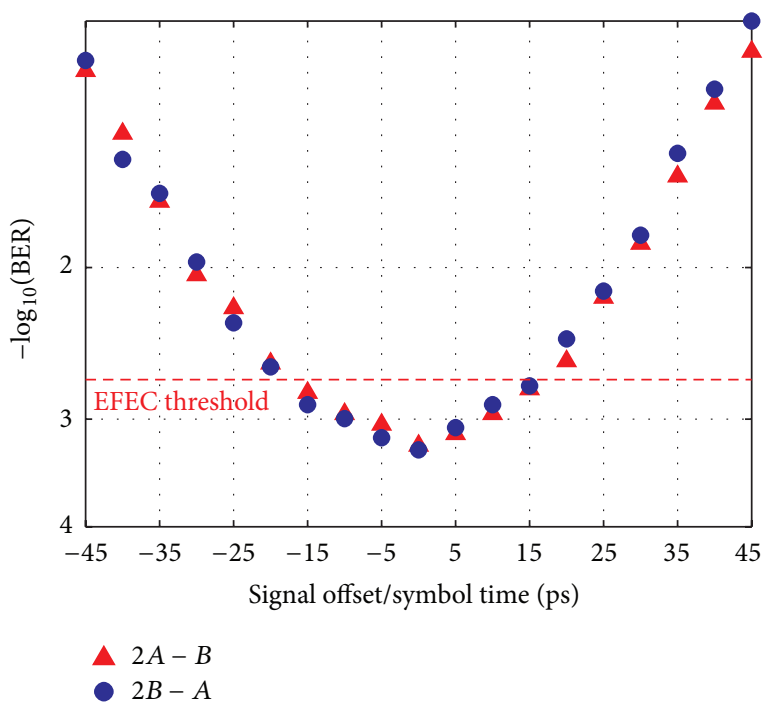

(b)

FIGURE 14: (a) Measured BER curves for two-input hybrid quaternary arithmetic functions of $2 A-B$ and $2 B-A$; (b) BER versus signal offset.

accumulated distortions transferred from two-input signals $(A, B)$. The main factors influencing the conversion efficiency can be briefly explained as follows. (1) In the experiment, only single-layer graphene is coated on the optical fiber pigtail cross section. So the weak light-graphene interaction could decrease the conversion efficiency [23]. With future improvement, one might improve the conversion efficiency by mechanically transferring graphene sample onto the $\mathrm{D}$ shaped fiber or microfiber to enhance the graphene-light interaction [17]. (2) The quality of graphene may also influence the conversion efficiency. Ideal graphene has unique dispersionlessness, broadband structure and strong third-order nonlinearity [12]. However, practically fabricated graphene is not perfect and any imperfections during the fabrication of 


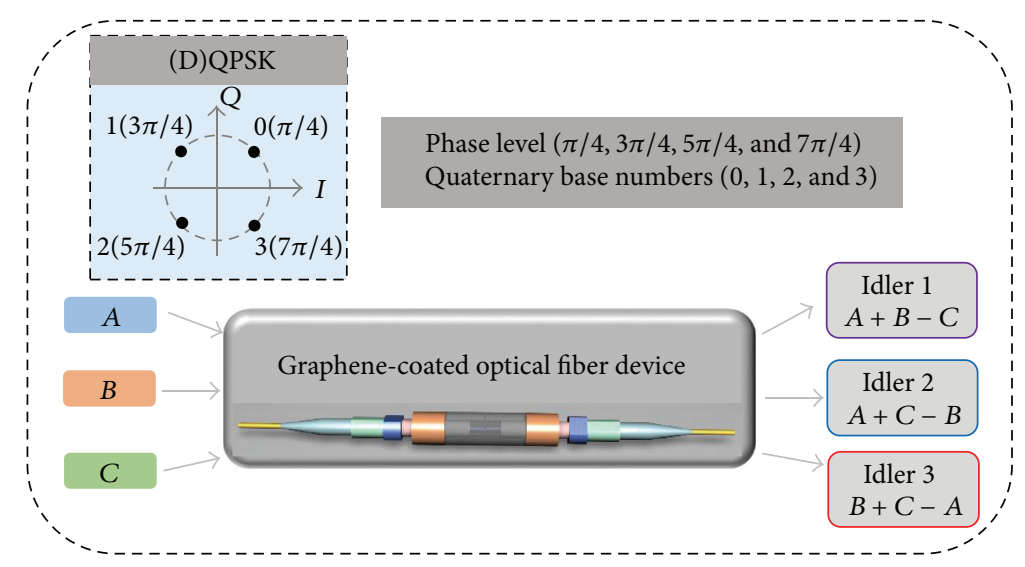

(a)



(b)

Figure 15: (a) Concept and (b) principle of graphene-assisted three-input $(A, B$, and $C)$ quaternary hybrid addition and subtraction $(A+B-C$, $A+C-B$, and $B+C-A$ ) using nondegenerate FWM and (D)QPSK signals.

graphene can break the band structure and degrades thirdorder nonlinearity. Thus the limited fabrication quality of the single-layer graphene employed in the experiment might also influence the conversion efficiency. (3) Previous work has demonstrated that the nonlinear response is also sensitive to the number of graphene layers [12]. It is expected that for a few graphene layers the nonlinearity increases in proportion to the number of layers. So it is possible to further enhance the conversion efficiency by appropriately increasing the number of graphene layers employed in the experiment. Figure 14(b) depicts the BER performance as a function of the relative time offset between two signals (signal offset) under an OSNR of $\sim 20 \mathrm{~dB}$. It is found that the BER is kept below enhanced forward error correction (EFEC) threshold when the signal offset/symbol time is within $15 \mathrm{ps}$, which indicates a favorable tolerance to the signal offset.

\section{Three-Input High-Base Optical Computing}

We also propose an approach to performing three-input optical addition and subtraction of quaternary base numbers using multiple nondegenerate FWM processes based on graphene-coated fiber device.
Figure 15 illustrates the concept and working principle of the proposed graphene-assisted three-input high-base optical computing, that is, optical addition and subtraction of quaternary base numbers. From the constellations in the complex plane (i.e., $I / Q$ plane), it is clear that we can use four-phase levels $(\pi / 4,3 \pi / 4,5 \pi / 4$, and $7 \pi / 4)$ of (D)QPSK to represent quaternary base numbers $(0,1,2$, and 3$)$. To implement three-input optical quaternary addition and subtraction, a single nonlinear device (e.g., graphene-coated optical fiber) is employed. Three-input (D)QPSK signals ( $A$, $B$, and $C$ ) are launched into the nonlinear device, in which three converted idlers (idler 1 , idler 2 , and idler 3 ) are simultaneously generated by three nondegenerate FWM processes. To better understand the working principle, we derive the electrical field $(E)$ and optical phase $(\Psi)$ relationships of three nondegenerate FWM processes under the no-depletion approximation expressed as

$$
\begin{aligned}
& E_{i 1} \propto E_{A} \cdot E_{B} \cdot E_{C}^{*}, \\
& \Psi_{i 1}=\Psi_{A}+\Psi_{B}-\Psi_{C}, \\
& E_{i 2} \propto E_{A} \cdot E_{C} \cdot E_{B}^{*},
\end{aligned}
$$




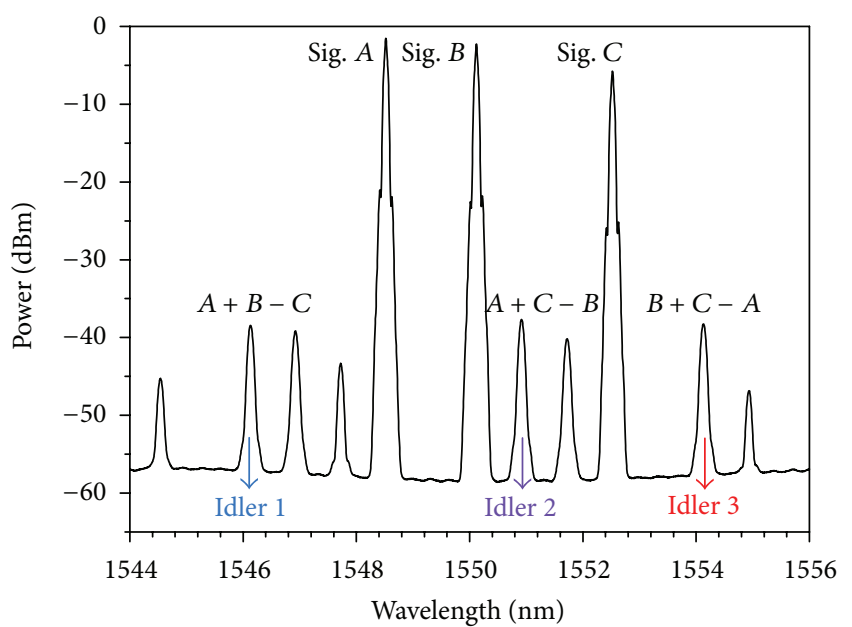

FIGURE 16: Measured spectrum for 10-Gbaud three-input quaternary hybrid addition and subtraction.

$$
\begin{aligned}
& \Psi_{i 2}=\Psi_{A}+\Psi_{C}-\Psi_{B}, \\
& E_{i 3} \propto E_{B} \cdot E_{C} \cdot E_{A}^{*}, \\
& \Psi_{i 3}=\Psi_{B}+\Psi_{C}-\Psi_{A},
\end{aligned}
$$

where the subscripts $A, B, C, i 1, i 2$, and $i 3$ denote input signal $A$, signal $B$, signal $C$, output idler 1 , idler 2 , and idler 3 , respectively. Considering the phase wrap characteristic with a period of $2 \pi$, the linear phase relationships in (4b), (5b), and (6b) imply that three converted idlers 1 3 correspond to modulo 4 operations of quaternary hybrid addition and subtraction of $A+B-C, A+C-B$, and $B+C-A$, respectively.

In the experiment, the wavelengths of three-input signals $A, B$, and $C$ are fixed at $1548.52,1550.12$, and $1552.52 \mathrm{~nm}$, respectively. Figure 16 depicts measured typical optical spectrum obtained after the single-layer graphene-coated fiber device. One can clearly see that three converted idlers are generated by three nondegenerate FWM processes with idler 1 at $1546.13 \mathrm{~nm}(A+B-C)$, idler 2 at $1550.92 \mathrm{~nm}(A+C-B)$, and idler 3 at $1554.13 \mathrm{~nm}(B+C-A)$, respectively. The power of HP-EDFA is estimated to be $31 \mathrm{dBm}$. The conversion efficiencies of three nondegenerate FWM processes are measured to be larger than $-34 \mathrm{~dB}$.

\section{High-Speed Gate-Tunable Terahertz Coherent Perfect Absorption [25]}

We present novel graphene split-ring metamaterials, which display a strong plasmonic resonance in the terahertz regime. First, by controlling the relative phase of the two coherent beams incident on the graphene film, we are able to change the absorption of graphene. Second, by introducing a proper chemical potential of graphene which can be controlled by means of applying a driven voltage, we can achieve gatetunable terahertz CPA. Finally, we analyze the tuning range of center frequency and the response time by using a resistorcapacitor (RC) circuit model.

In the principle of CPA [37], manipulating either the phase or intensity of beam B modulates the transmitted intensity of beam A. The coherent absorption can be tuned by changing the relative phase of two beams, which has recently been reported in nanostructured graphene film [38], nonresonant suspending monolayer graphene [39], unstructured multilayer graphene films [40], and planar metamaterials [37]. To optimize the modulation efficiency, the graphene film should absorb half of the energy of a single beam passing through it, which can be realized based on the split-ring resonator structure. Figure 17 shows the 2D and 3D structures of the proposed split-ring graphene film for CPA. For splitring graphene film with symmetric two-side (air, air) conditions (Case 1), as illustrated in Figure 17(a), the interference of two counterpropagating incident beams $\mathrm{A}$ and $\mathrm{B}$ (relative intensity $I_{\mathrm{A}} / I_{\mathrm{B}}=1$ ) on such a film is described by two special cases. When the phase difference $\varphi_{\mathrm{A}}-\varphi_{\mathrm{B}}=0$, the CPA occurs. So the graphene reaches the maximum absorption. When the phase difference $\varphi_{\mathrm{A}}-\varphi_{\mathrm{B}}=\pi$, beam $\mathrm{A}$ and beam $B$ pass though one another without mutual disturbance. For split-ring graphene film with asymmetric two-side (air, $\mathrm{SiO}_{2}$ ) conditions (Case 2), gate-tunable CPA is illustrated in Figure 17(b) which is based on the electroabsorption effect of graphene. By electrically tuning the Fermi level of the graphene sheet, corresponding to chemical potential $\mu_{c 1}$, the absorption of split-ring graphene reaches the maximum limit $\sim 50 \%$ for single incident beam. In this situation, when two coherent beams with equal intensities and phases are incident on the graphene from opposite sides, they will interfere with each other, leading to coherent absorption. When the chemical potential of graphene is changed to $\mu_{c 2}$, the absorption of split-ring graphene is tuned to be weak. So, beam $A$ and beam $B$ pass though one another nearly without loss. Hence, gate-tunable CPA is achievable. The geometric parameters of the patterned split-ring graphene are shown in Figure 17 (c). The period is $L=20 \mu \mathrm{m}$ and the radii of inner and outer circle are $r_{1}=5 \mu \mathrm{m}$ and $r_{2}=8 \mu \mathrm{m}$, respectively. The gap aperture of the split-ring is $d=2 \mu \mathrm{m}$.

We first study the CPA of Case 1 (Figure 17(a)). The CPA is enabled by $50 \%$ absorption of single beam and equal intensities and phases of two counterpropagating coherent beams. The chemical potential (Fermi energy) $\mu_{c}=0.3 \mathrm{eV}$ and relaxation time $\tau=0.5$ ps are initially considered. The transmission $(T)$, reflection $(R)$, and absorption $(A)$ of a split-ring graphene patterned with periodical array are plotted in Figure 18(a). When a single beam is illuminated on the patterned split-ring graphene film at normal incidence, one can see an obvious resonance around $2.91 \mathrm{THz}$. The strong resonance behaviors are expected to be electric dipolar mode. The excitation of electric dipolar mode results in the enhancement of absorption in the graphene sheet with a maximum of $A=49.92 \%$ while the other parts of the incident beam energy are reflected or transmitted. As shown in Figure 18(b), when two coherent beams with equal intensities and phases are incident on the graphene from opposite sides, they will interfere with each other, leading to coherent absorption of $99.69 \%$. 


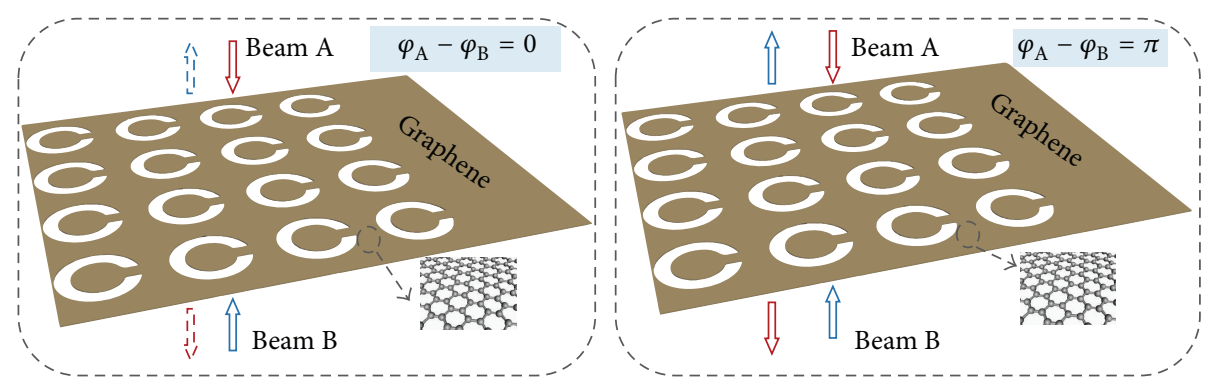

(a)



(b)

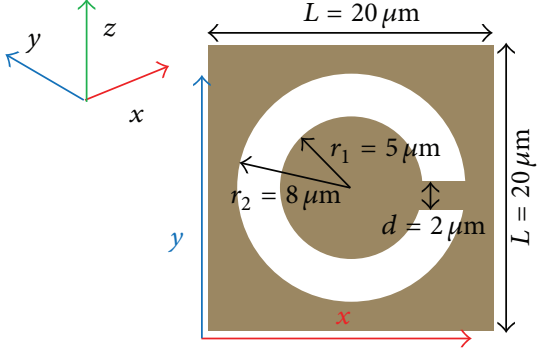

(c)

Figure 17: (a) Schematic of coherent perfect absorption in a split-ring graphene film (Case 1). Two coherent optical beams (A, B) impinge on the graphene film from opposite sides at normal incidence. (b) Interaction of light with light on a split-ring graphene $\mathrm{SiO}_{2}$ substrate (Case 2). (c) A unit cell of the split-ring graphene film with geometric parameters. The period is $L=20 \mu \mathrm{m}$ and the radii of inner and outer circle are $r_{1}=5 \mu \mathrm{m}, r_{2}=8 \mu \mathrm{m}$, respectively. The gap aperture $(d)$ of the split-ring is $2 \mu \mathrm{m}$.



(a)

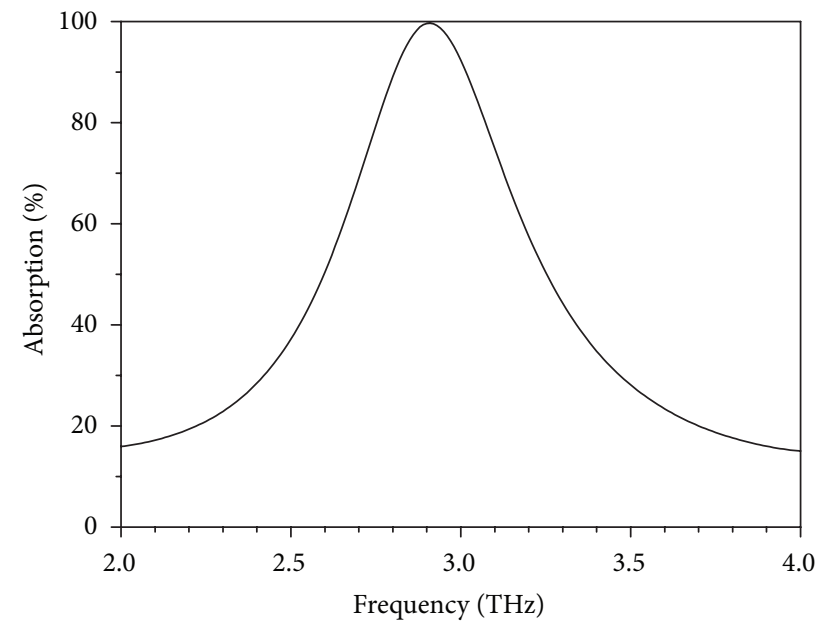

(b)

Figure 18: (a) Calculated $T, R$, and $A$ of the split-ring graphene film under the illumination of only one beam at normal incidence. (b) Normalized total absorption under the illumination of two counterpropagating coherent beams with the same intensities and phases. The chemical potential of graphene is assumed to be $\mu_{c}=0.3 \mathrm{eV}$.

Figure 19(a) shows the normalized coherent absorption in the split-ring graphene as a function of the relative phase different between two counterpropagating coherent beams. At the resonance frequency of $2.91 \mathrm{THz}$, the coherent absorption varies continuously from $99.7 \%$ to less than $2.1 \times 10^{-4} \%$ as the phase difference changes from 0 to $0.969 \pi$, giving a modulation contrast of $56.7 \mathrm{~dB}$. In practical fabrication processes, the geometric parameters of the split-ring might be slightly offset from their designed values. So it is valuable to comprehensively study the impacts of these possible practical 


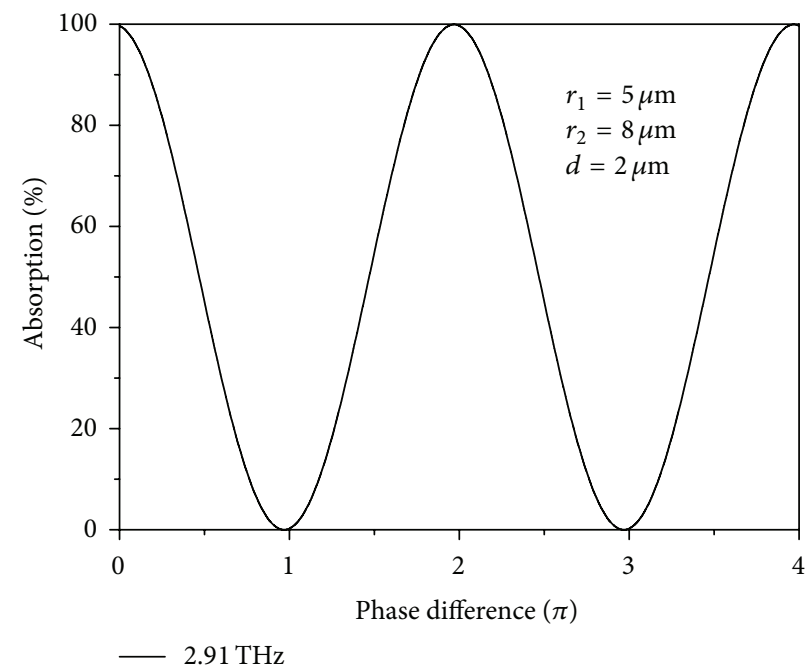

(a)



(c)

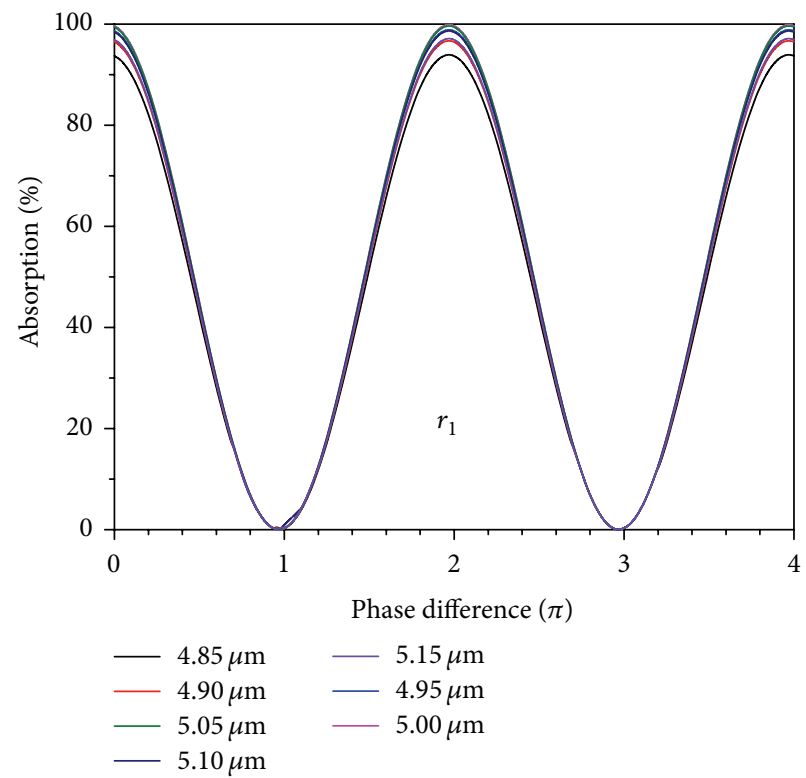

(b)



(d)

FIGURE 19: (a) Calculated normalized total absorption versus relative phase difference between two counterpropagating coherent beams. (b)-(d) The tolerance of the geometric parameters $\left(r_{1}, r_{2}\right.$, and $d$ ) on the device performance (absorption). Case 1.

fabrication imperfections on the operation performance of graphene split-ring resonators, as shown in Figures 19(b), 19(c), and 19(d).

We then study the gate-tunable CPA of Case 2 (Figure $17(\mathrm{~b})$ ). The gate-tunable operation relies on the electroabsorption effect of graphene. We consider the same splitring graphene film on a $\mathrm{SiO}_{2}$ substrate with a refractive index of $n=1.45$, as shown in Figure 17(b). Figures 20(a) and 20(b) present calculated normalized absorption spectra for single-beam surface-normal illumination from the split-ring graphene side and the $\mathrm{SiO}_{2}$ side, respectively, under different chemical potentials (Fermi energies). By electrically tuning the chemical potential of the split-ring graphene sheet, one can change the resonance center frequency. For illumination from the split-ring graphene side, the maximum absorption keeps $\geq 49.5 \%$ when the center frequency varies from 2.29 to $2.71 \mathrm{THz}$, as shown in Figure 20(a). For illumination from the $\mathrm{SiO}_{2}$ substrate side, the maximum absorption keeps $\geq 49.8 \%$, as shown in Figure 20(b). Figure 21 displays the calculated total absorption spectra of the split-ring graphene under different chemical potentials. An absorption peak of 97.13\% at $2.29 \mathrm{THz}$ is seen from Figure 21(a) for $\mu_{c}=0.24 \mathrm{eV}$. 


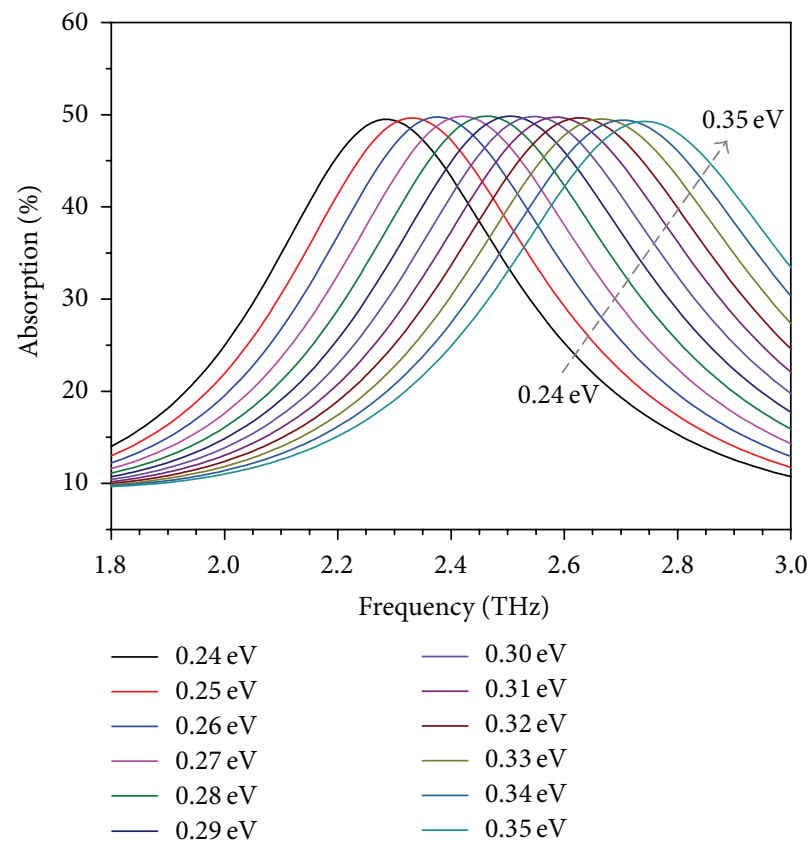

(a)

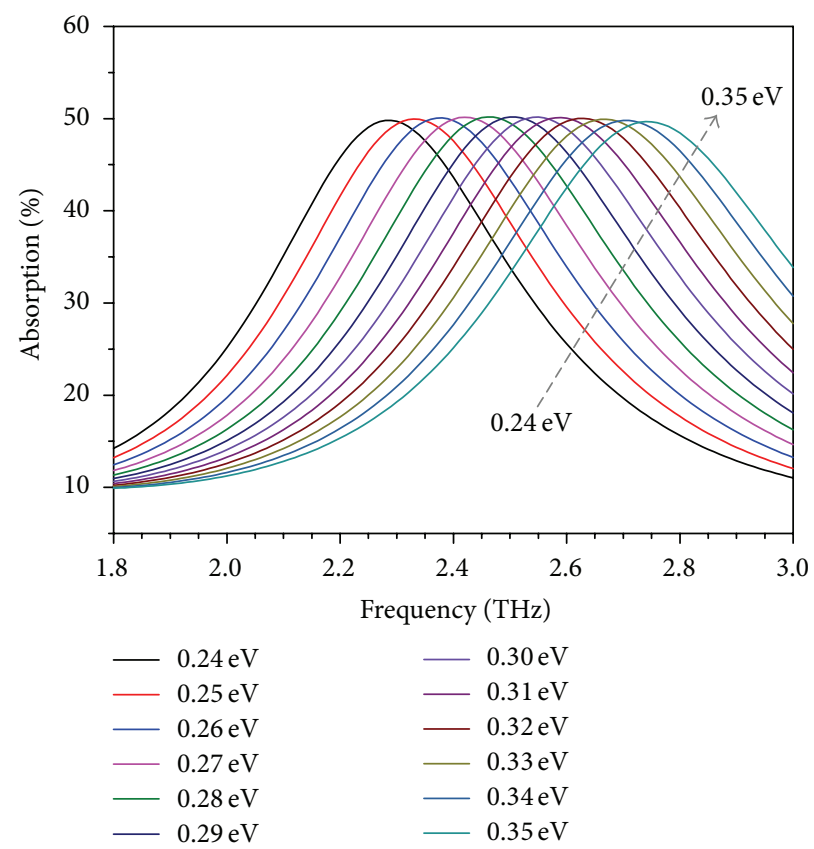

(b)

FIGURE 20: Calculated normalized absorption spectra for single-beam surface-normal illumination from (a) split-ring graphene side and (b) $\mathrm{SiO}_{2}$ side under different chemical potentials of graphene. Case 2 .



(a)

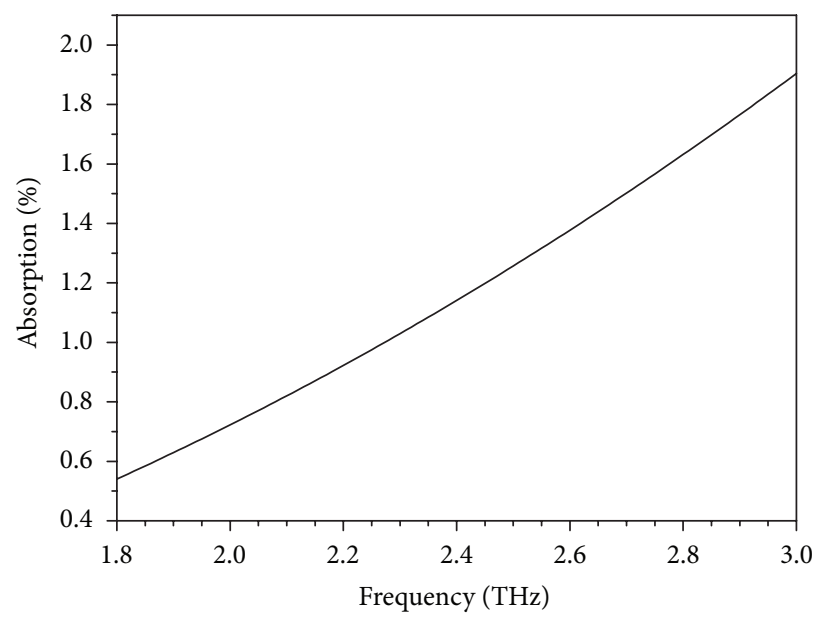

(b)

Figure 21: (a) Center frequency tunable total absorption spectra of the split-ring graphene under different chemical potentials. (b) Total absorption of the split-ring graphene with chemical potential $\mu_{c}=0 \mathrm{eV}$. Case 2 .

With the increase of the graphene chemical potential, it shows a blue shift of the resonance peak frequency. When the chemical potential of graphene is tuned to $\mu_{c}=0.35 \mathrm{eV}$, the absorption at $2.71 \mathrm{THz}$ can still reach $95.6 \%$. As the Fermi level of graphene can be electrically tuned, one can flexibly control the total absorption of the split-ring graphene. The split-ring graphene possesses the minimum absorption when the chemical potential is $\mu_{c}=0 \mathrm{eV}$, as shown in Figure 21(b). For the center frequency at $2.5 \mathrm{THz}$, the maximum and minimum absorption are $97.5 \%\left(\mu_{c}=2.9 \mathrm{eV}\right)$ and 


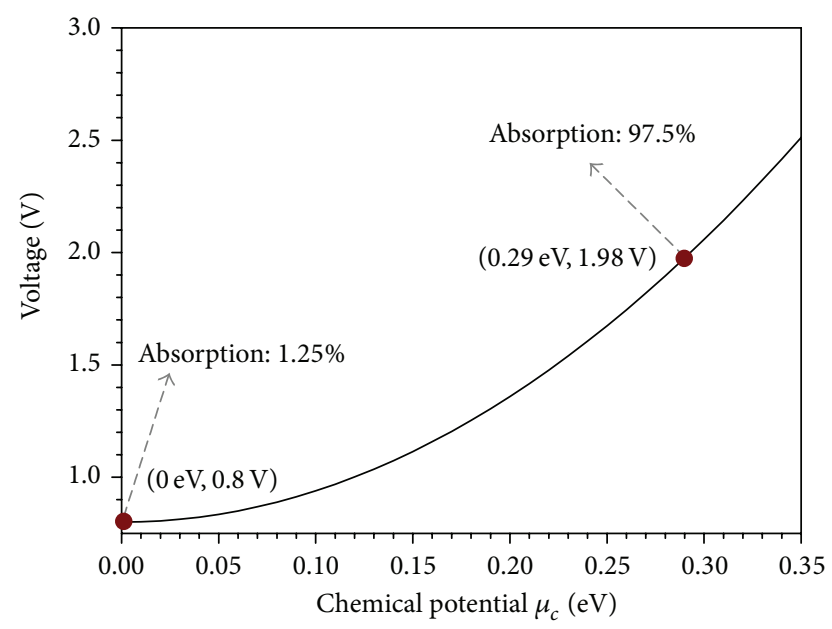

FIGURE 22: Driven voltage versus the chemical potential of graphene.

$1.25 \%\left(\mu_{c}=0 \mathrm{eV}\right)$, giving a gate-tunable modulation contrast of $19 \mathrm{~dB}$.

In the designed device (Figure 17(b)), the dielectric $\mathrm{SiO}_{2}$ strip sandwiched by graphene and Au forms a simple parallel capacitor model. The chemical potential $\mu_{c}$ of graphene can be tuned by applying a voltage which follows the relationship of $\mu_{c}=\hbar \nu_{F} \sqrt{\pi\left|\alpha\left(V_{g}+V_{0}\right)\right|}$ [5], where $\nu_{F}=3.0 \times 10^{6} \mathrm{~ms}^{-1}$ [41] is the Fermi velocity, $V_{0}$ is the voltage offset caused by the natural doping, and $V_{g}$ stands for the applied voltage. $\alpha=\varepsilon_{0} \varepsilon_{\text {die }} /$ de is estimated by using a parallel-plate capacitor, where $\varepsilon_{\text {die }}, d$, and $e$ represent the permittivity of the dielectric inside the capacitor, the distance between the capacitor plates, and the charge of an electron, respectively. Figure 22 shows the driven voltage versus the chemical potential of graphene. From the obtained results shown in Figures 20-22, one can see that gate-tunable CPA operation is achievable. By changing the driven voltage applied to graphene, it is possible to switch the absorption between $1.25 \%(0.8 \mathrm{~V})$ and $95.7 \%(1.98 \mathrm{~V})$ at $2.5 \mathrm{THz}$, implying a $\mathrm{THz}$ wave modulator. Meanwhile, center frequency tunable CPA is also achievable (Figure 21(a)).

The graphene-assisted device may possess fast response time (RT). With a carrier mobility exceeding $1,000,000 \mathrm{~cm}^{-2} \mathrm{~V}^{-1} \mathrm{~s}^{-1}$ at room temperature [42] and high saturation velocity of graphene, the operation speed of the device is not likely limited by the carrier transition time but $R_{m} C$ of the circuit, where $R_{m}$ and $C$ are the total series resistance and capacitance of the device, respectively. In the designed device, the total series resistance $R_{m}$ is calculated by adding the Ohmic resistances of graphene layer as well as the resistance $R_{c}$ of the metal contacts to the graphene layer; the former can be reduced to $\sim 125 \Omega$ /sq by highly doped regions, while the latter is several ohms [43]. We calculate the RT by the $R C$ constant of the device. The capacitance of the device can be calculated as $C=\varepsilon_{0} \varepsilon_{d} A_{\text {gra }} / d$, where $\varepsilon_{0}, \varepsilon_{d}, A_{\text {gra }}$, and $d$ represent the permittivity of vacuum, the permittivity of the dielectric inside the capacitor, the area of graphene, the distance between the capacitor plates, and the charge of an electron, respectively. The capacitance of the structure is calculated to be $C=1.8 \times 10^{-13} \mathrm{~F}$. According to RT $=R_{m} C$ [44], we can calculate the response time to be $\sim 36$ ps by taking $R_{m} \sim 20 \Omega$ [43], which indicates possible high-speed gate-tunable $\mathrm{THz}$ CPA operations.

\section{Discussions and Conclusions}

Looking back on the previous works of graphene-assisted nonlinear optical signal processing, Hendry and coworkers experimentally demonstrated the graphene based FWM for the first time in [12]. They also indicated that graphene might have large $\chi^{(3)}$ nonlinearity due to its linear band structure allowing interband optical transitions at all photon energies. After that, FWM was also observed in graphenesilicon hybrid optoelectronic devices [14] and graphenecoated microfiber $[17,18]$. Moreover, FWM based wavelength conversion of a $10-\mathrm{Gb} / \mathrm{s}$ NRZ signal with mechanically exfoliated graphene was first reported in [19].

In this paper, we have reviewed our recent progress in graphene-assisted nonlinear optical device and their applications, including degenerate FWM based tunable wavelength conversion of QPSK signal, phase conjugated wavelength conversion by degenerate FWM and transparent wavelength conversion by nondegenerate FWM, two-input optical highbase hybrid doubling and subtraction functions, three-input high-base optical computing, and high-speed gate-tunable terahertz coherent perfect absorption using a split-ring graphene.

For FWM based tunable wavelength conversion of QPSK signal, the total effective nonlinear Kerr coefficient of graphene-assisted nonlinear optical device is actually the combined contributions from the graphene and the device material (e.g., silica in graphene-coated fiber). It has to be emphasized that spectrally efficient advanced modulation formats have been widely used in optical fiber transmission systems. The advanced optical modulation formats play an important role in enabling high-capacity optical transport networks where wavelength conversion function is highly desired. Therefore, exploring wavelength conversion of advanced modulation formats based on FWM in graphene is very interesting and meaningful.

For phase conjugated and transparent wavelength conversions, the Nyquist 16-QAM signal is employed. Additionally, we demonstrated that, for the wavelength conversion based on degenerate FWM process, the newly converted idler does not take the same data information carried by the original signal but its "phase conjugated" copy, while for nondegenerate FWM process the newly converted idler copies exactly the same data information carried by the original signal, that is, fully transparent wavelength conversion.

For graphene-assisted optical computing (two-input and three-input), the innovative schemes to achieve hybrid quaternary arithmetic functions of doubling and subtraction using optical nonlinearities in graphene and (D)QPSK signals are presented. By exploiting degenerate FWM in graphene, we experimentally demonstrate 10 -Gbaud quaternary arithmetic functions of $2 A-B$ and $2 B-A$. The power penalties of converted idlers at a BER of $2 \times 10^{-3}$ are measured to be about $7.4 \mathrm{~dB}$ for $2 A-B$ and $7.0 \mathrm{~dB}$ for $2 B-A$. 
TABLE 1: Comparison among silica, silicon, and graphene.

\begin{tabular}{lcccr}
\hline Platforms & Kerr coefficient & Loss & Compactness & Compatibility $^{*}$ \\
\hline Silica in fiber [21] & $\sim 10^{-20} \mathrm{~m}^{2} / \mathrm{W}$ & Low & Large footprint & Fully compatible \\
Silicon [22] & $\sim 10^{-18} \mathrm{~m}^{2} / \mathrm{W}$ & High & Compact & Incompatible \\
Graphene-coated fiber [13] & $\sim 10^{-11} \mathrm{~m}^{2} / \mathrm{W}$ & Low & Compact & Fully compatible \\
\hline
\end{tabular}

${ }^{*}$ Compatibility of silica in fiber, silicon photonic device, and graphene-assisted nonlinear optical fiber devices with existing optical fiber transmission systems.

By exploiting nondegenerate FWM in graphene, we demonstrate 10 -Gbaud three-input hybrid addition and subtraction of quaternary base numbers $(A+B-C, A+C-B$, and $B+C-A)$. With future improvement, graphene-assisted nonlinear optical devices might be employed to facilitate more optical signal processing applications.

For high-speed gate-tunable terahertz CPA using a splitring graphene, the designed structure has benefits of tunable center frequency, ultrafast response time, small footprints, ease of fabrication, and compatibility with standard complementary metal-oxide semiconductor (CMOS) processing $[45,46]$. With future improvement, the proposed high-speed gate-tunable CPA using split-ring graphene may find interesting applications in modulators, detectors, and sensors.

Actually, there are different platforms for nonlinear optical signal processing, for example, silica in fiber, silicon, and graphene. Table 1 summarizes a brief comparison among silica in fiber, silicon, and graphene. The Kerr coefficients of silica in fiber, silicon, and graphene are $\sim 10^{-20} \mathrm{~m}^{2} / \mathrm{W}$, $\sim 10^{-18} \mathrm{~m}^{2} / \mathrm{W}$, and $\sim 10^{-11} \mathrm{~m}^{2} / \mathrm{W}$, respectively. Nonlinear optical signal processing based on silica in fiber has lower power loss. Although silica in fiber is compatible with optical fiber transmission systems, its third-order nonlinearity is lower and the desired fiber is longer (i.e., larger footprint). Silicon has higher $\chi^{(3)}$ nonlinearity and the compactness of silicon photonic device is suitable for chip-scale optical signal processing functions. However, silicon photonic device is not compatible with optical fiber transmission systems. In contrast, graphene has even larger $\chi^{(3)}$ nonlinearity and the fabricated graphene-assisted nonlinear optical fiber device with the graphene placed within the connector of two fibers is fully compatible with existing optical fiber transmission systems. The $\chi^{(3)}$ nonlinearity of graphene is several orders of magnitude larger than silica in fiber and silicon, which is due to the unique linear band structure of $\pi$-electrons $[12,47,48]$. The graphene-assisted nonlinear optical fiber device is compact. The combined effective nonlinearity of graphene-assisted nonlinear optical fiber device is increased and the graphene enhances the FWM process. However, the measured conversion efficiency of graphene-assisted nonlinear optical fiber device in the experiment is $\sim 33 \mathrm{~dB}$, which is lower than highly nonlinear fiber. It is noted that practically fabricated graphene is not perfect and any imperfections during the fabrication of graphene can break the band structure and degrades the dispersionlessness and $\chi^{(3)}$ nonlinearity properties. Additionally, FWM response (conversion efficiency) is also dependent on the number of graphene layers. Previous work demonstrated that the nonlinear response was sensitive to the number of graphene layers [12]. It is expected that for a few graphene layers the nonlinearity increases in proportion to the number of layers, suggesting the tremendous potential of graphene as a platform for efficient nonlinear optical signal processing. In this scenario, it is possible to further enhance the FWM response by appropriately increasing the number of graphene layers employed in the experiment.

\section{Competing Interests}

The authors declare that they have no competing interests.

\section{Acknowledgments}

This work was supported by the National Natural Science Foundation of China (NSFC) under Grants nos. 61222502, 11574001, and 11274131, the National Basic Research Program of China (973 Program) under Grant no. 2014CB340004, the Program for New Century Excellent Talents in University (NCET-11-0182), the Wuhan Science and Technology Plan Project under Grant no. 2014070404010201, and the seed project of Wuhan National Laboratory for Optoelectronics (WNLO). The authors thank the engineer in the Center of Micro-Fabrication and Characterization (CMFC) of WNLO for the support in the fabrication of graphene-assisted nonlinear optical device and the facility support of the Center for Nanoscale Characterization and Devices of WNLO.

\section{References}

[1] K. S. Novoselov, A. K. Geim, S. V. Morozov et al., "Electric field in atomically thin carbon films," Science, vol. 306, no. 5696, pp. 666-669, 2004.

[2] K. Kim, J.-Y. Choi, T. Kim, S.-H. Cho, and H.-J. Chung, "A role for graphene in silicon-based semiconductor devices," Nature, vol. 479, no. 7373, pp. 338-344, 2011.

[3] F. Xia, T. Mueller, Y.-M. Lin, A. Valdes-Garcia, and P. Avouris, "Ultrafast graphene photodetector," Nature Nanotechnology, vol. 4, no. 12, pp. 839-843, 2009.

[4] Q. Bao, H. Zhang, B. Wang et al., "Broadband graphene polarizer," Nature Photonics, vol. 5, no. 7, pp. 411-415, 2011.

[5] M. Liu, X. Yin, E. Ulin-Avila et al., "A graphene-based broadband optical modulator," Nature, vol. 474, no. 7349, pp. 64-67, 2011.

[6] A. N. Grigorenko, M. Polini, and K. S. Novoselov, "Graphene plasmonics," Nature Photonics, vol. 6, no. 11, pp. 749-758, 2012.

[7] Q. Bao, H. Zhang, Y. Wang et al., "Atomic-layer craphene as a saturable absorber for ultrafast pulsed lasers," Advanced Functional Materials, vol. 19, no. 19, pp. 3077-3083, 2009. 
[8] Z. Sun, T. Hasan, F. Torrisi et al., "Graphene mode-locked ultrafast laser," ACS Nano, vol. 4, no. 2, pp. 803-810, 2010.

[9] Q. Bao, H. Zhang, Z. Ni et al., "Monolayer graphene as a saturable absorber in a mode-locked laser," Nano Research, vol. 4, no. 3, pp. 297-307, 2011.

[10] Y. Meng, S. Zhang, X. Li, H. Li, J. Du, and Y. Hao, "Multiplesoliton dynamic patterns in a graphene mode-locked fiber laser," Optics Express, vol. 20, no. 6, pp. 6685-6692, 2012.

[11] N. Tolstik, E. Sorokin, and I. T. Sorokina, "Graphene modelocked Cr:ZnS laser with $41 \mathrm{fs}$ pulse duration," Optics Express, vol. 22, no. 5, pp. 5564-5571, 2014.

[12] E. Hendry, P. J. Hale, J. Moger, A. K. Savchenko, and S. A. Mikhailov, "Coherent nonlinear optical response of graphene," Physical Review Letters, vol. 105, no. 9, Article ID 097401, 2010.

[13] H. Zhang, S. Virally, Q. Bao et al., "Z-scan measurement of the nonlinear refractive index of graphene," Optics Letters, vol. 37, no. 11, pp. 1856-1858, 2012.

[14] T. Gu, N. Petrone, J. F. McMillan et al., "Regenerative oscillation and four-wave mixing in graphene optoelectronics," Nature Photonics, vol. 6, no. 8, pp. 554-559, 2012.

[15] H. Zhou, T. Gu, J. F. McMillan et al., "Enhanced four-wave mixing in graphene-silicon slow-light photonic crystal waveguides," Applied Physics Letters, vol. 105, no. 9, Article ID 091111, 2014.

[16] B. Xu, A. Martinez, K. Fuse, and S. Yamashita, "Generation of four wave mixing in graphene and carbon nanotubes optically deposited onto fiber ferrules," in Proceedings of the Conference on Lasers and Electro-Optics (CLEO '11), pp. 1-2, Baltimore, Md, USA, May 2011.

[17] Y. Wu, B. Yao, Y. Cheng et al., "Four-wave mixing in a microfiber attached onto a graphene film," IEEE Photonics Technology Letters, vol. 26, no. 3, pp. 249-252, 2014.

[18] Y. Wu, B. C. Yao, Q. Y. Feng et al., "Generation of cascaded four-wave-mixing with graphene-coated microfiber," Photonics Research, vol. 3, no. 2, pp. A64-A68, 2015.

[19] B. Xu, A. Martinez, and S. Yamashita, "Mechanically exfoliated graphene for four-wave-mixing-based wavelength conversion," IEEE Photonics Technology Letters, vol. 24, no. 20, pp. 1792-1794, 2012.

[20] P. J. Winzer and R.-J. Essiambre, "Advanced modulation formats for high-capacity optical transport networks," Journal of Lightwave Technology, vol. 24, no. 12, pp. 4711-4728, 2006.

[21] L. Prigent and J.-P. Hamaide, "Measurement of fiber nonlinear kerr coefficient by four-wave mixing," IEEE Photonics Technology Letters, vol. 5, no. 9, pp. 1092-1095, 1993.

[22] M. Dinu, F. Quochi, and H. Garcia, "Third-order nonlinearities in silicon at telecom wavelengths," Applied Physics Letters, vol. 82, no. 18, pp. 2954-2956, 2003.

[23] X. Hu, M. Zeng, A. Wang, L. Zhu, L. Fu, and J. Wang, "Graphene-assisted nonlinear optical device for four-wave mixing based tunable wavelength conversion of QPSK signal," Optics Express, vol. 23, no. 20, pp. 26158-26167, 2015.

[24] A. Wang, X. Hu, L. Zhu, M. Zeng, L. Fu, and J. Wang, "Experimental demonstration on two-input optical high-base hybrid doubling and subtraction functions in graphene," Optics Express, vol. 23, no. 25, pp. 31728-31735, 2015.

[25] X. Hu and J. Wang, "High-speed gate-tunable terahertz coherent perfect absorption using a split-ring graphene," Optics Letters, vol. 40, no. 23, pp. 5538-5541, 2015.

[26] F. Bonaccorso, Z. Sun, T. Hasan, and A. C. Ferrari, "Graphene photonics and optoelectronics," Nature Photonics, vol. 4, no. 9, pp. 611-622, 2010.
[27] Q. Bao and K. P. Loh, "Graphene photonics, plasmonics, and broadband optoelectronic devices," ACS Nano, vol. 6, no. 5, pp. 3677-3694, 2012.

[28] B. Sensale-Rodriguez, R. Yan, M. M. Kelly et al., "Broadband graphene terahertz modulators enabled by intraband transitions," Nature Communications, vol. 3, article 780, 2012.

[29] M. S. Kwon, "Discussion of the epsilon-near-zero effect of graphene in a horizontal slot waveguide," IEEE Photonics Journal, vol. 6 , no. 3, pp. 1-9, 2014.

[30] F. H. L. Koppens, D. E. Chang, and F. J. García de Abajo, "Graphene plasmonics: a platform for strong light-matter interactions," Nano Letters, vol. 11, no. 8, pp. 3370-3377, 2011.

[31] L. A. Falkovsky and S. S. Pershoguba, "Optical far-infrared properties of a graphene monolayer and multilayer," Physical Review B, vol. 76, no. 15, Article ID 153410, 2007.

[32] L. A. Falkovsky and A. A. Varlamov, "Space-time dispersion of graphene conductivity," The European Physical Journal B, vol. 56, no. 4, pp. 281-284, 2007.

[33] S. Ke, B. Wang, H. Huang, H. Long, K. Wang, and P. Lu, "Plasmonic absorption enhancement in periodic cross-shaped graphene arrays," Optics Express, vol. 23, no. 7, pp. 8888-8900, 2015.

[34] X. Li, W. Cai, J. An et al., "Large-area synthesis of high-quality and uniform graphene films on copper foils," Science, vol. 324, no. 5932, pp. 1312-1314, 2009.

[35] A. C. Ferrari, J. C. Meyer, V. Scardaci et al., "Raman spectrum of graphene and graphene layers," Physical Review Letters, vol. 97, no. 18, Article ID 187401, 2006.

[36] L. M. Malard, M. A. Pimenta, G. Dresselhaus, and M. S. Dresselhaus, "Raman spectroscopy in graphene," Physics Reports, vol. 473, no. 5-6, pp. 51-87, 2009.

[37] J. Zhang, K. F. MacDonald, and N. I. Zheludev, "Controlling light-with-light without nonlinearity," Light: Science \& Applications, vol. 1, no. 18, article e18, 2012.

[38] J. Zhang, C. Guo, K. Liu et al., "Coherent perfect absorption and transparency in a nanostructured graphene film," Optics Express, vol. 22, no. 10, pp. 12524-12532, 2014.

[39] Y. Fan, F. Zhang, Q. Zhao, Z. Wei, and H. Li, “Tunable terahertz coherent perfect absorption in a monolayer graphene," Optics Letters, vol. 39, no. 21, pp. 6269-6272, 2014.

[40] S. M. Rao, J. J. F. Heitz, T. Roger, N. Westerberg, and D. Faccio, "Coherent control of light interaction with graphene," Optics Letters, vol. 39, no. 18, pp. 5345-5347, 2014.

[41] R. Hao, W. Du, H. Chen, X. Jin, L. Yang, and E. Li, "Ultracompact optical modulator by graphene induced electrorefraction effect," Applied Physics Letters, vol. 103, no. 6, Article ID 061116, 2013.

[42] J. Baringhaus, M. Ruan, F. Edler et al., "Exceptional ballistic transport in epitaxial graphene nanoribbons," Nature, vol. 506, no. 7488, pp. 349-354, 2014.

[43] C. Qiu, W. Gao, R. Vajtai, P. M. Ajayan, J. Kono, and Q. Xu, "Efficient modulation of $1.55 \mu \mathrm{m}$ radiation with gated graphene on a silicon microring resonator," Nano Letters, vol. 14, no. 12, pp. 6811-6815, 2014.

[44] A. Melikyan, N. Lindenmann, S. Walheim et al., "Surface plasmon polariton absorption modulator," Optics Express, vol. 19, no. 9, pp. 8855-8869, 2011.

[45] M. Liu, X. B. Yin, and X. Zhang, "Double-layer graphene optical modulator," Nano Letters, vol. 12, no. 3, pp. 1482-1485, 2012.

[46] A. Pospischil, M. Humer, M. M. Furchi et al., "CMOS-compatible graphene photodetector covering all optical communication bands," Nature Photonics, vol. 7, no. 11, pp. 892-896, 2013. 
[47] A. K. Geim and K. S. Novoselov, "The rise of graphene," Nature Materials, vol. 6, no. 3, pp. 183-191, 2007.

[48] K. S. Novoselov, A. K. Geim, S. V. Morozov et al., "Two-dimensional gas of massless Dirac fermions in graphene," Nature, vol. 438, no. 7065, pp. 197-200, 2005. 

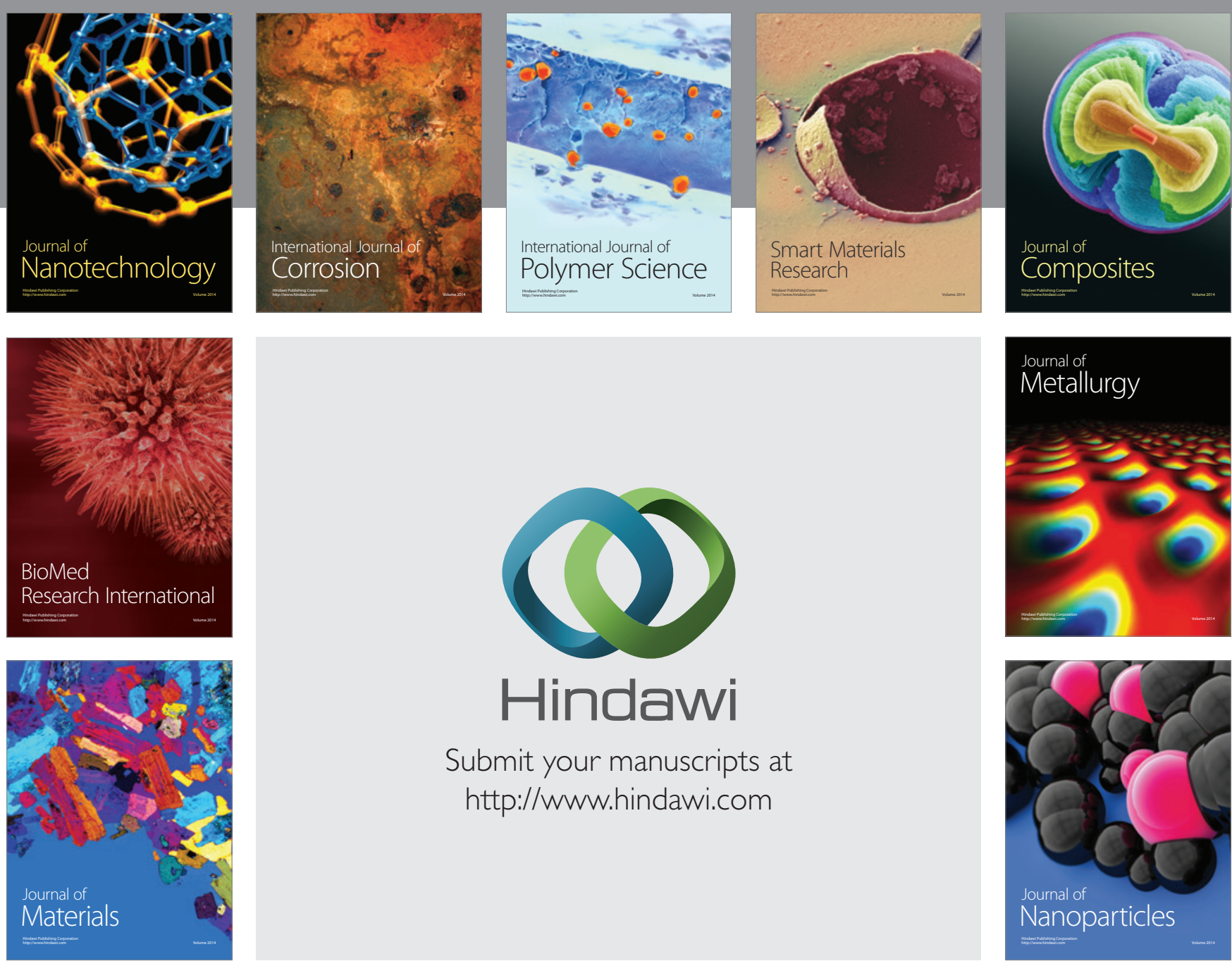

\section{Hindawi}

Submit your manuscripts at

http://www.hindawi.com

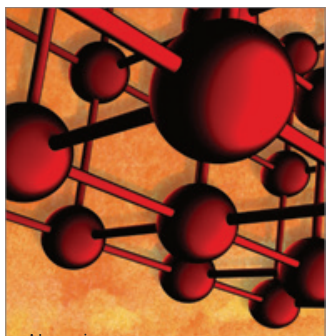

Materials Science and Engineering
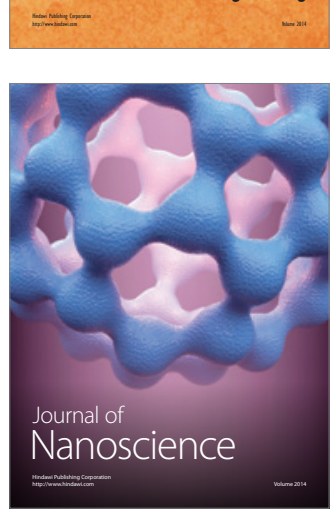

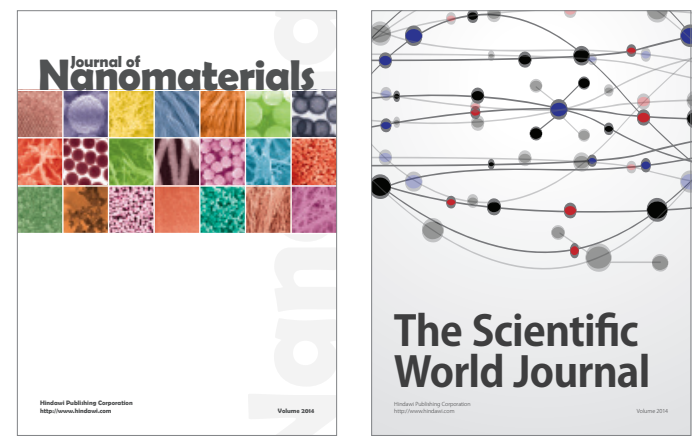

The Scientific World Journal
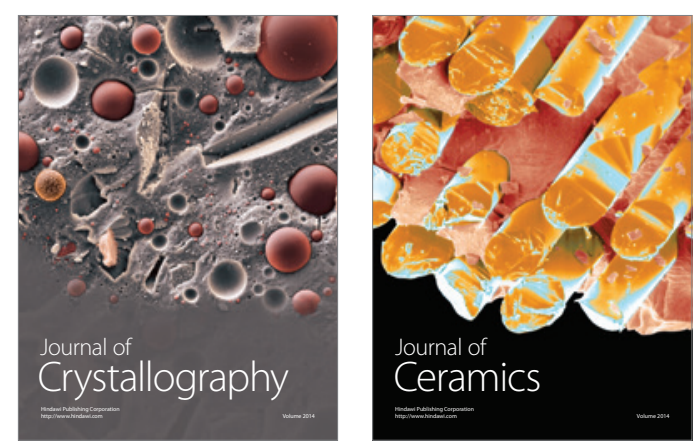
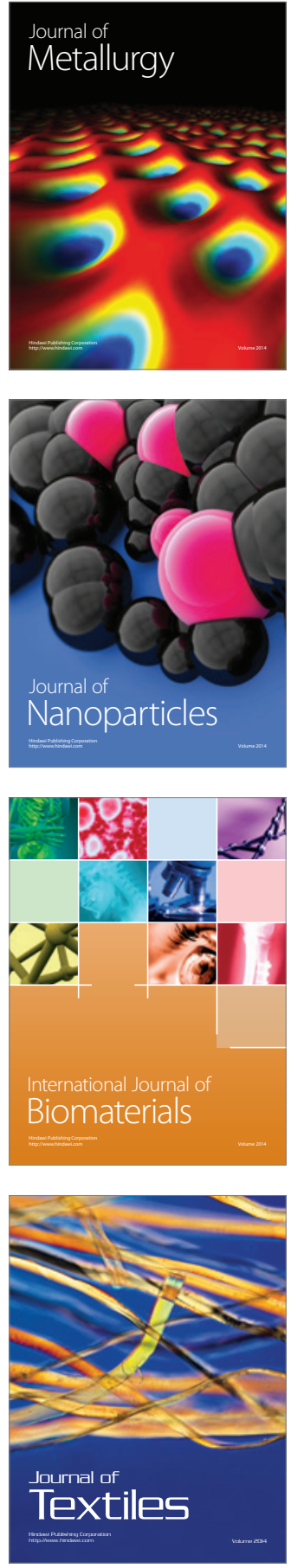